\title{
4 Swallowed by Lions and Eagles. Das AFSC in der Sowjetunion 1921-1923
}

Im Sommer 1921 war Herbert Hoovers Geduld mit den Quäkern an ein Ende gelangt. Er schlug mit der Faust auf den Tisch und fluchte dabei wie ein Bierkutscher (,like a trooper“). Seit einigen Wochen bereitete die ARA unter seiner Führung eine weitere große Hilfsaktion vor - diesmal in der noch jungen Sowjetunion. In den laufenden Verhandlungen mit den Bolschewiki hatte er zuvor immer wieder einen Satz zu hören bekommen: „Why don’t you work like the Quakers do?“1 Für Hoover brachte dies das Fass zum Überlaufen. Nun stellte er Wilbur Thomas ein Ultimatum: Das AFSC würde in Russland Teil einer ARA-Hilfsaktion werden - zu seinen Bedingungen und unter seiner Führung - oder er werde dafür Sorge tragen, dass die Quäker dort keinen Fuß auf den Boden bekämen.

Wenngleich die Episode sich am Ende als Sturm im Wasserglas entpuppen sollte: Sie wirft ein Schlaglicht auf den politisch hochbrisanten Charakter der anlaufenden Hilfsaktion in der jungen Sowjetunion, ${ }^{2}$ bei der sich das AFSC ab Sommer 1921 erneut an der Seite der ARA wiederfand. ${ }^{3}$ Der Anlass war eine Hungersnot, die ein Land traf, das von sieben Jahren Krieg, Flucht und Bürgerkrieg bereits ausgezehrt war, als ab Ende Mai die Hauptgetreideanbaugebiete Russlands, zwischen Wolga und Don, von einer Dürre heimgesucht wurden. Nach Schätzungen waren bis zu 20 Millionen Menschen unmittelbar vom Hungertod

1 Wilbur Thomas an Rufus Jones 18/1/1923, in Rufus Jones Papers, Haverford College Quaker Collection, zitiert nach David McFadden and Claire Gorfinkel, Constructive Spirit: Quakers in Revolutionary Russia (Pasadena: Intentional Productions, 2004), 63.

2 Im folgenden Kapitel verwende ich zuweilen den Begriff „Sowjetunion“ für das, was für den Großteil des Einsatzes korrekter als „Sowjetrussland“ beziehungsweise als „Russische SFSR“ bezeichnet werden müsste. Die offizielle Gründung der Sowjetunion erfolgte als Zusammenschluss verschiedener Teilrepubliken im Dezember 1922.

3 Zur Quäkerhilfsaktion und auch den internen Spannungen, die sie begleiteten ist David McFadden und Claire Gorfinkels Band Constructive Spirit. Quakers in Revolutionary Russia sehr hilfreich. Er enthält neben einer Sammlung von Quellen und Kurzzusammenfassungen zu einzelnen Aspekten der Hilfsoperation auch eine (von Sergei Nikitin verfasste) ausführliche Chronologie der Aktion vor Ort McFadden and Gorfinkel, Constructive Spirit: Quakers in Revolutionary Russia. Einen Überblick über die Hilfsaktion in der SU aus verschiedenen Perspektiven mit Fokus auf die „moral economy“ der Kampagnen westlicher Hilfsorganisationen bieten: Götz, Brewis, and Werther, Humanitarianism in the Modern World: The Moral Economy of Famine Relief. Hier firmiert der russische Fall neben der irischen Hungersnot von 1847/48 und der Äthiopiens in den 1980er Jahren als archetypischer Fall eines Einsatzes im Zeitalter des „organized humanitarianism“.

Ә OpenAccess. (C 2022 Daniel Maul, publiziert von De Gruyter. (cc) BY-NC-ND Dieses Werk ist lizenziert unter einer Creative Commons Namensnennung - 4.0 International Lizenz.

https://doi.org/10.1515/9783110675788-006 
bedroht. ${ }^{4}$ Vor diesem Hintergrund wurde das AFSC ab Herbst 1921 zum zweiten Mal Teil einer großen Hilfsoperation, die rein äußerlich einige Ähnlichkeiten zu dem parallel laufenden deutschen Fall aufwies: Auch in Russland wurde das AFSC Teil einer ARA-Operation, ${ }^{5}$ welche die Auflösung einer bestehenden britisch-amerikanischen Hilfsmission erforderte. Noch dringlicher als in Deutschland stellte sich in Russland die Frage der politischen Neutralität der Quäker beziehungsweise der Loyalität des AFSC gegenüber den Zielen der amerikanischen Außenpolitik (und ihrem inoffiziellen Vertreter Hoover). Schmerzhafter als in Deutschland sollten sich die Quäker in Russland dem Dilemma ausgesetzt sehen, den quäkerspezifischen Gehalt ihres Einsatzes in Einklang mit den praktischen Anforderungen einer großangelegten Hilfsoperation $\mathrm{zu}$ bringen. In weitaus höherem Maße als in Deutschland löste der russische Einsatz sowohl innerhalb des Komitees als auch zwischen dem home office in Philadelphia und den Helfern vor Ort eine politische Debatte um die Position des Komitees aus, die zu einer Zerreißprobe für das AFSC führte. ${ }^{6}$

\section{Die Quäker in Russland}

Als die Nachrichten von der Dürre in den Kornkammern Russlands und der drohenden Hungersnot die USA im Sommer 1921 erreichten, wurde dies im AFSC als unmittelbarer Aufruf zur Aktion verstanden. Dies mag auf den ersten Blick verwunderlich erscheinen angesichts der Tatsache, dass die Hilfsaktion in Deutschland zu diesem Zeitpunkt einen gehörigen Teil der Kapazitäten der Quäkerhilfe in Anspruch nahm. Bei näherer Betrachtung war die schnelle Reaktion jedoch durchaus logisch, denn als einzige US-amerikanische Hilfsorganisation unterhielt das AFSC zu diesem Zeitpunkt bereits eine kleine Hilfsmission in der Sowjetunion.

Vor allem britische Quäker hatten seit dem 19. Jahrhundert enge Kontakte nach Russland. Britische und US-amerikanische Quäker spielten eine Rolle bei der Umsiedlung der im Zarenreich verfolgten Dukhobor-Sekte nach Kanada, einer

\footnotetext{
4 Eine reflektierte Einschätzung zu den Zahlen bei Rodney Breen, „Saving Enemy Children: Save the Children's Russian Relief Operation, 1921-1923“, in: Disasters 18, 3 (1994), 221-238.

5 Siehe hierzu die umfassende Studie von Patenaude, The Big Show in Bololand: The American Relief Expedition to Soviet Russia in the Famine of 1921. Für einen knappen Überblick mit Fokus auf die Person Hoovers Bruno Cabanes, The Great War and the Origins of Humanitarianism, 1918-1924 (Cambridge: Cambridge University Press, 2014), 189-247.

6 McFadden and Gorfinkel, Constructive Spirit: Quakers in Revolutionary Russia; McFadden, „The Poltics of Relief: American Quakers and Russian Bolsheviks, 1917-1921.“
} 
Gruppe von religiösen Dissenters. ${ }^{7}$ Um die Wende zum 20. Jahrhundert kamen die Verbindungen vor allem britischer Friends zum pazifistischen und lebensreformerischen Kreis der „Tolstoianer“ hinzu. ${ }^{8}$ Diese Kontakte bildeten den Hintergrund für die Beteiligung der Quäker an ersten Hilfseinsätzen in Russland während der großen Hungersnöte 1891/92 und 1906, die sich überwiegend in Samara und der Kreisstadt Buzuluk in der Wolgaregion konzentrierten - auch danach das Haupteinsatzgebiet der Quäkerhilfe in der Sowjetunion. ${ }^{9}$

Die Verbindungen wurden während des Ersten Weltkriegs zunächst von den britischen Quäkern reaktiviert. Im April 1916 entsandte das Friends War Victims Relief Committee (FWVRC) eine Delegation unter der Leitung von Joseph Burtt, einem der führenden britischen Tolstoianer, in die Wolgaregion. Ihm folgte eine erste Gruppe von rund 40 Helfern, die im August ihre Arbeit unter Leitung des Neuseeländers Theodore Rigg für die in Buzuluk ankommenden Flüchtlingsströme von der polnischen Front aufnahmen. Viele von ihnen teilten Erfahrungen aus Quäkersanitätseinheiten an anderen Fronten des Krieges. ${ }^{10}$ Nach dem amerikanischen Kriegseintritt stieß eine Gruppe von sechs Frauen aus den USA zur britischen Einheit, unter ihnen mit Anna Haines, Nancy Babb und Esther White drei der prägenden Figuren des AFSC-Einsatzes in Russland in der ersten Hälfte der 1920er Jahre. ${ }^{11}$

Ein Artikel im AFSC-Bulletin von Mitte 1918 warb um Spenden für Russland und bilanzierte das erste Jahr der amerikanischen Teilnahme an der Unternehmung. Er zeichnete das Bild eines Einsatzes, der trotz der wachsenden Anzahl von Helfern rasch über die Möglichkeiten der Quäkermission hinauswuchs - bedingt durch das schiere Ausmaß des Flüchtlingselends auf einem Gebiet, das in etwa der Größe Belgiens entsprach. ${ }^{12}$ Die politischen Umstürze des Jahres 1917 hatten

7 Charlotte Alston, ,'A Great Host of Sympathisers': The Doukhobor Emigration and Its International Supporters, 1895-1905,“ Journal of Modern European History 12, 2 (2014).

8 Greenwood, Quaker Encounters Vol. 1: Friends and Relief, 124.

9 Zum Einsatz britischer Gruppen in Russland siehe Luke Kelly, British Humanitarian Activity in Russia, 1890-1923 (Cham: Springer, 2018). Zu den USA Curti, American Philanthropy Abroad, $259-300$.

10 In der Kreisstadt Buzuluk machten zu diesem Zeitpunkt Flüchtlinge mit ca. 26.000 Personen rund ein Viertel der Gesamtbevölkerung aus. William Cadbury, Joseph Burtt, Robert Tatlock und Theodore Rigg besaßen Erfahrungen aus ihren Einsätzen in Quäkersanitätseinheiten in Frankreich, Albanien und Montenegro.

11 Die übrigen Mitglieder dieser Gruppe waren Emilie Bradbury, Lydia Lewis und Amelia Fabriszewski.

12 Die Quäkereinrichtungen - ein Hospital, ein Waisenhaus und fünf Arbeitszentren für Flüchtlinge - konnten dem stetigen Zustrom von Flüchtlingen in der Region kaum standhalten, 
kaum unmittelbar negative Folgen für die Hilfsmission. Trotz der Oktoberrevolution, des beginnenden Bürgerkriegs sowie der Landung alliierter Truppen in Sibirien, ging die Arbeit in Buzuluk zunächst weiter. Parallel reisten Theodore Rigg und Esther White nach Moskau, um die Möglichkeiten für die Einrichtung eines Quäkerzentrums auszuloten. Im September schlossen die britischen und amerikanischen Friends einen Vertrag mit den Sowjetbehörden, der den Quäkern zunächst die Sorge für den Unterhalt dreier Kolonien für Waisenkinder in Tambov sowie in der Gegend von Woronesch übertrug. Damit verbunden, gestatteten die Behörden die Öffnung eines Quäkerbüros in Moskau, das als Liaison zur Sowjetregierung sowie als Verbindungsbüro zwischen den home offices in Philadelphia und London und den Missionen vor Ort dienen sollte. Dieses Büro, deren erste Leiterin Esther White wurde, bildete den Ausgangspunkt einer Quäker-Repräsentanz in der Sowjetunion, die mit kurzen Unterbrechungen bis Ende der 1920er Jahre weiterbestand. ${ }^{13}$

Zeitgleich mit der Eröffnung des Moskauer Büros verschlechterten sich die Bedingungen für die Hilfsarbeit in Buzuluk rapide. Im August kam es zum endgültigen Abbruch der Beziehungen zwischen der Sowjetregierung und den westlichen Mächten, die im Bürgerkrieg offen Partei für die anti-bolschewistische Seite zu ergreifen begannen. Nachdem Buzuluk im Juni 1918 von tschechoslowakischen Truppen eingenommen worden war, um im September erneut an die Bolschewiki zu fallen, musste die Mission schließlich aufgelöst werden. Die meisten Helfer kehrten nach England und in die USA zurück, andere arbeiteten für das Amerikanische Rote Kreuz und die YMCA auf von „Weißer“ Seite kontrolliertem Gebiet. Auch für Rigg und White wurde die Lage in Moskau schließlich zu unsicher. Beide verließen Russland im Februar 1919 über den Hafen von Riga, während Anna Haines über Wladiwostok in die USA zurückkehrte. ${ }^{14}$

Wenngleich die Fronten zwischen Russland und dem Westen damit zunehmend verhärtet schienen ${ }^{15}$, begannen die Quäker unmittelbar nach der Ausreise

zumal die Mission in zunehmendem Maße akute Hungerhilfe zu leisten hatte. „The Bitter Cry of Russia“, in: AFSC-Bulletin 16 (1918).

13 Als Ansprechpartner dienten Rigg und White dabei die Mitglieder der philanthropischen Pirogovtsi-Gesellschaft, einem Kreis von Tolstoianern, der in Moskau vier Waisenhäuser unterhielt. Sergei Nikitin, A Personal overview in: McFadden and Gorfinkel, Constructive Spirit: Quakers in Revolutionary Russia, 11-12.

14 Sergei Nikitin Sergei Nikitin, A Personal overview in: McFadden and Gorfinkel, Constructive Spirit: Quakers in Revolutionary Russia, 12.

15 Während die Sowjetregierung ausländischen Besitz enteignete, hielt der Westen eine vollständige Handelsblockade gegen die Bolschewiki aufrecht. Weiterhin standen an mehreren Orten innerhalb Russlands westliche Interventionstruppen bereit. US-amerikanische Truppen sicherten 
der letzten Helfer bereits mit den Planungen für eine Rückkehr. Besonders für amerikanische Hilfsorganisationen war es jedoch von diesem Zeitpunkt an schwierig, auf von den Bolschewiki gehaltenem Territorium tätig zu werden. Eine britisch-amerikanische Delegation von Quäkern hatte sich am Rande der Pariser Friedensverhandlungen vergeblich bemüht, die Unterstützung ihrer Regierungen für einen Hilfseinsatz zu erhalten. Ihr Ansprechpartner auf US-Seite war auch in diesem Fall Herbert Hoover gewesen, der sich dem Anliegen gegenüber zunächst aufgeschlossen zeigte - in der vergeblichen Hoffnung, im Gegenzug die Bolschewiki zu Konzessionen bewegen zu können. ${ }^{16}$ Frankreich und Großbritannien waren ihrerseits kategorisch gegen einen solchen Einsatz unter Beteiligung amerikanischer Quäker, nicht zuletzt ,because they suspect American commercial aspirations Russia“, wie ein Mitglied der Delegation telegrafisch zu berichten wusste. Unbeeindruckt von den Widerständen war man in Philadelphia dennoch weiter entschlossen, ,to keep on the lookout for any opportunity to press our way into Russia“. ${ }^{17}$

Unterstrichen wurde dieses Ziel durch die Einrichtung eines Russian Committee 1919, das unter der Leitung von Henry Cadbury arbeitete. Eine erste Öffnung erfolgte zu Beginn des Jahres 1920, als sich einerseits der Sieg der Roten Armee im Bürgerkrieg abzuzeichnen begann, während parallel der Ausbruch des polnisch-russischen Krieges im April 1920 zu einer weiteren Verschlechterung der Versorgungslage führte. Vor diesem Hintergrund zeigte sich die Sowjetregierung offener für westliche Hilfsangebote. Im Juni reisten zunächst zwei britische Quäker, Arthur Watts und Gregory Welch, nach Moskau, um Möglichkeiten einer Wiederaufnahme direkter Hilfsleistungen in Buzuluk auszuloten. Ende 1920 folgte dann auch Anna Haines für das AFSC, die gemeinsam mit Watts die Leitung des Moskauer Quäkerbüros übernahm. Beide schlossen ein Abkommen mit dem Volkskommissariat für Nahrungsmittelproduktion (Narkomprod) zur Lagerung,

den Hafen von Wladiwostok als Versorgungsposten für die anti-bolschewistische Seite. Briten und Franzosen hatten kleinere Kontingente in Sibirien und auf der Krim im Einsatz.

16 Hoover fürchtete zudem, dass ohne ein Abkommen amerikanische Staatsbürger den Bolschewiki als Geiseln dienen könnten. In einem Gespräch, das eine Quäkerdelegation mit Fridtjof Nansen führte, umriss dieser Hoovers Strategie wie folgt: „Mr. Hoover's hope is that by feeding and supporting all the governments around Russia, the Bolshevik regime can be kept behind a certain line, that it must agree if it is to be fed, to stay behind that line." Hoover selbst beschreibe seinen Plan „as a bribe of food to get the Bolshevik to stop fighting on our side of the line“. Der Bericht über das Gespräch bei J. H. Scattergood an Ruth Fry 26/4/1919, in: LRSF, Box 7, Panel 5 Folder 1: Russia, Reports, Correspondence etc.

17 Das Russian Committee des AFSC unter Leitung von Henry Cadbury bekräftigte kurze Zeit später seine Intention, wieder aktiv zu werden, 22/5/1919, in: AFSCA, AFSC Minutes 1919. 
Lieferung und Verteilung von Hilfsgütern in Russland und erhielten Büros und Lagerräume von der russischen Zentrale für Kooperativen. ${ }^{18}$

Zur selben Zeit blieb die Lage für das AFSC selbst aber prekär. Im April 1921 bat Wilbur Thomas in einem Schreiben an Außenkommissar Georgi Tschitscherin um die Erlaubnis, zusätzliches Personal nach Russland entsenden zu können. ${ }^{19}$ In seinem Antwortschreiben stellte Tschitscherin dem Friends International Service die förmliche Einladung aus, welche die Quäker berechtigte, nicht nur eigene Hilfsgüter, sondern auch Sendungen anderer Organisationen wie Save the Children und sogar solche des Amerikanischen Roten Kreuzes zu verteilen. Damit verbunden war auch die Erlaubnis, die Mission mit amerikanischen Quäkern zu besetzen. Zur selben Zeit machte Tschitscherin jedoch klar, dass die Erlaubnis nicht zu einer Öffnung Sowjetrusslands führen dürfe ,for all kind of foreign relief work societies and organizations that are desirous of doing relief work here“. Die Mehrheit dieser Organisationen sei „unfriendly towards the Soviet government and will not fail to utilize every possibility in order to make their activities a source for all kind of intrigues and anti-Soviet propaganda“. ${ }^{20}$

Für das AFSC bedeutete diese Regelung die Möglichkeit, sowohl Gelder als im Prinzip auch Personal nach Russland zu senden. Entscheidend war in letzter Hinsicht allerdings, dass die Erlaubnis für die Quäker als „,people who have really proven their absolute abstinence from politics“, nicht jedoch für deren nationale Hilfsorganisationen galt. ${ }^{21}$ Die letzte Entscheidung über die Einreiseerlaubnis für amerikanische Hilfsarbeiter wurde durch die Sowjetbehörden somit weiter auf individueller Basis getroffen. In der Praxis wurde diese Politik bis in den Sommer

18 Vor diesem Hintergrund wurde das AFSC durch das State Department darüber unterrichtet, dass einer erneuten Einreise von Quäkerhelfern nach Russland, freilich ohne diplomatischen Schutz, keine grundsätzlichen Hindernisse im Weg stünden. Im selben Monat erhielten die Quäker die Erlaubnis, Hilfsgüter nach Russland einzuführen, zunächst unter der Maßgabe, dass ihre Verteilung in der Obhut der Sowjetbehörden verblieb. in: AFSCA, AFSC Minutes 1919.

19 Wilbur Thomas an Georgi Tschitscherin (Volkskommissar für Auswärtige Angelegenheiten) 7/ 4/1921, in: AFSCA, General Administration, Foreign Service, Country: Russia 1921 (letters from ARA, Russian Unit).

20 Georgi Tschitscherin an Arthur Watts 14/4/1921, in: AFSCA, General Administration, Foreign Service, Country: Russia 1921 (letters to Philadelphia from Russia).

21 Tschitscherin führte aus: „Moreover we cannot agree with the principle that the relief organizations of the very same capitalistic countries which thru their blockade are strangling millions of Russian workers, their wives and children should attempt with their offered assistance to mask and cover the most horrible features of the inhuman policy of their countries. The possible assistance that can be rendered even in cases where the desires prompting are absolutely sincere is read at best to but buv very little and cannot bring about any serious positive results." Georgi Tschitscherin an Arthur Watts 14/4/1921, in: AFSCA, General Administration, Foreign Service, Country: Russia 1921 (letters to Philadelphia from Russia). 
äußerst restriktiv gehandhabt. Während damit im Frühjahr und selbst im Sommer 1921 nur wenige amerikanische Quäker tatsächlich in Russland präsent waren, war das AFSC aktiv bemüht um sowohl die Finanzierung als auch die Vorbereitung einer vergrößerten Hilfsaktion. Im April reiste Anna Haines nach Buzuluk, um die Wiederaufnahme der direkten Hilfsleistungen vorzubereiten. Im Mai und Juni handelte Haines mit den lokalen Behörden die Erlaubnis zum Aufbau einer Hilfsmission in der Region aus. $\mathrm{Zu}$ diesem Zeitpunkt verdichteten sich bereits überall die Vorzeichen, dass der Region und anderen wichtigen Getreideanbaugebieten in Russland und der Ukraine aufgrund einer anhaltenden Trockenheit eine Hungersnot bevorstand, die selbst die Zustände während der vorangegangenen Kriegs- und Bürgerkriegsjahre in den Schatten zu stellen drohte. ${ }^{22}$

\section{Hoover reloaded. Das AFSC und der ARA-Einsatz in Russland 1921}

An dieser Stelle trat Herbert Hoover auf den Plan. Nur wenige Tage nachdem der bekannte russische Schriftsteller Maxim Gorki (unter offensichtlicher Billigung der Sowjetregierung) im Juli einen Appell „an alle wohlmeinenden Völker“ gerichtet hatte, ${ }^{23}$ unterrichtete Hoover das AFSC über seine Absicht, eine Hilfsaktion unter Federführung der ARA zu organisieren. Dem AFSC als Partner in einer solchen Kampagne stellte Hoover dabei eine gewaltige Menge an Hilfsgütern in Aussicht. ${ }^{24}$ Äußerlich glichen die Voraussetzungen damit jenen am Ausgangspunkt der Kinderspeisungsaktion in Deutschland eineinhalb Jahre zuvor. Im Unterschied zum deutschen Fall war Hoovers Initiative diesmal nur eine unter mehreren teils miteinander konkurrierenden internationalen Initiativen die auf den Gorki-Aufruf folgten. ${ }^{25}$

Anfang August organisierte das Internationale Rote Kreuz mit Unterstützung des Genfer Völkerbundes eine Konferenz privater Hilfsorganisationen aus 22 Ländern, an der auch eine Reihe von Regierungen meist kleinerer Länder teilnahm. Aus dem Treffen ging das International Committee for Russian Relief

22 Anna Haines an Wilbur Thomas 14/7/1921, in: AFSCA, General Administration, Foreign Service, Country: Russia 1921 (letters to Philadelphia from Russia).

23 Das Telegramm im englischen Wortlaut bei McFadden and Gorfinkel, Constructive Spirit: Quakers in Revolutionary Russia, 59.

24 Hoover an Thomas 13/7/1921, in: AFSCA, General Administration, Comms and Orgs 1921 (American Relief Administration).

25 Maul, „Appell an Das Gewissen Der Völker - Fridtjof Nansen, Der Internationale Humanitarismus und die russische Hungerhilfe 1921-1923“. 
(ICRR) hervor, als dessen Hoher Kommissar der Norweger Fridtjof Nansen diente. Nansen hatte 1920 im Auftrag des Völkerbundes den Austausch von Hunderttausenden seit dem Weltkrieg internierten Kriegsgefangenen zwischen Russland und dem Deutschen Reich sowie den Nachfolgestaaten der Donaumonarchie koordiniert und verfügte über gute Kontakte nach Moskau. ${ }^{26}$ Gegen das von Nansen verfolgte Ziel, das Hilfsprogramm durch Kredite der europäischen Mächte an die Sowjetunion zu finanzieren, stand in den meisten europäischen Ländern, vor allem in Großbritannien und Frankreich, eine starke öffentliche Meinung. Schnell wurde deutlich, dass die Nansen-Mission kaum auf offizielle Unterstützung zählen konnte und zum großen Teil über private Spenden finanziert werden musste. Die Hoffnungen richteten sich damit auf die USA und namentlich auf Hoover, der jedoch ganz andere Pläne verfolgte.

$\mathrm{Zu}$ einer Aktion in Zusammenarbeit oder gar unter der Ägide des IRK oder des Völkerbundes ${ }^{27}$ war Hoover nicht bereit. Vielmehr wollte er die Russlandhilfe wie in Deutschland als rein amerikanisches Unternehmen und unter eigenen Prämissen durchführen. Seine Motive ähnelten denen, die der Hilfe in Deutschland zugrunde lagen. Grundsätzlich war er davon überzeugt, dass Hunger die Voraussetzung für den Erfolg des bolschewistischen Regimes darstellte. Wenn es der ARA gelänge, die Hungerhilfe im direkten Kontakt mit der russischen Bevölkerung zu organisieren, würde diesem System auf zweifache Weise die Grundlage entzogen: Die Beseitigung des Hungers und die Begegnung mit den überlegenen Ressourcen und Methoden der USA würden als quasi natürliche Konsequenz zum Verschwinden des Bolschewismus führen.

Hoovers Anti-Bolschewismus und sein Glaube an die Überlegenheit des kapitalistischen Systems verband sich im russischen Fall darüber hinaus mit handfesten wirtschaftlichen Interessen: Hoover sah Russland sowohl kurzfristig mit Bezug auf die Hilfsaktion als auch langfristig als einen Absatzmarkt für den

\footnotetext{
26 Das Berliner Büro, über das der Austausch hauptsächlich organisiert wurde (die operationelle Durchführung lag in der Hand des Internationalen Roten Kreuzes), firmierte als „Nansen-Hilfe“. Zur Aktion Martyn Housden, „When the Baltic Sea was a ,Bridge for Humanitarian Action“: The League of Nations, the Red Cross and the Repatriation of Prisoners of War between Russia and Central Europe, 1920 -22“, in: Journal of Baltic Studies 38, 1 (2007), 61-63; speziell zu Nansens Rolle siehe Carl Emil Vogt, Nansens Kamp Mot Hungersnøden I Russland 1921-1923 (Oslo: Aschehoug, 2007); Carl Emil Vogt, „Fridtjof Nansen and European Food Aid to Russia and the Ukraine 1921-1923,“ The Twentieth Century/Dvacáte století, 2 (2009).

27 Die USA waren kein Mitglied des Völkerbundes. Auch die Beziehungen zum Roten Kreuz waren durch die Gegensätze zwischen dem Genfer Internationalen Rotkreuzkomitee (ICRC) und der vom ARC dominierten International League of Red Cross Societies getrübt. Dieter Riesenberger, Für Humanität in Krieg Und Frieden: Das Internationale Rote Kreuz 1863-1977 (Göttingen: Vandenhoeck \& Ruprecht, 1992).
} 
Überschuss der US-Agrarindustrie. Humanitäre, diplomatische und außenwirtschaftliche Ziele waren somit aus Hoovers Sicht eins. ${ }^{28}$ Im Gegensatz zum NansenKomitee gebot die ARA über ein Vielfaches an Mitteln sowie über eine unmittelbar mobilisierbare Hilfsmaschinerie, was ihr von Anfang an eine deutlich bessere Verhandlungsposition gab. Bereits am 20. August (und damit eine Woche vor Nansen und dem International Committee) schloss Hoover in der lettischen Hauptstadt Riga ein Abkommen mit der Sowjetregierung, welches der ARA weitgehend freie Hand bei der Durchführung der Hilfsaktion in Russland verschaffte. Die Sowjetregierung verpflichtete sich im Gegenzug zur Bezahlung eines Teils der Hilfsgüter mittels russischer Goldreserven. ${ }^{29}$

Diese Initiative brachte für das AFSC unmittelbar die Frage nach dem Verhältnis zur vorgesehenen ARA-Operation auf den Tisch. Dies nicht nur aufgrund der gleichzeitigen Zusammenarbeit in Deutschland. Vielmehr blickten AFSC und ARA auch in Russland bereits auf eine längere gemeinsame Geschichte zurück. Dabei war es zunächst das AFSC gewesen, das sich seit Sommer 1920 beständig um Hoovers Unterstützung für die Hilfsaktionen in Buzuluk und Moskau bemüht hatte. Streitpunkte zwischen beiden Parteien war dabei stets gewesen, inwieweit das AFSC im Gegenzug bereit war, US-amerikanische Interessen gegenüber der Sowjetregierung wahrzunehmen, und inwieweit die Quäker garantieren konnten, dass ihr Geld nicht in den Händen der Bolschewiki enden würde.

Hoover hatte dem AFSC gleichwohl Anfang 1921 seine Unterstützung in Aussicht gestellt, falls die Quäker bei der Sowjetregierung die Freilassung amerikanischer Kriegsgefangener (aus dem Bürgerkrieg) erwirkten. Wilbur Thomas hatte dies seinerzeit mit der Begründung abgelehnt, dass eine solche Aktion die Quäker als offizielle Agenten amerikanischer Interessen hätte erscheinen lassen. Auch vor diesem Hintergrund hatte sich Thomas seit Anfang 1921 um Alternativen bei der Finanzierung der Russlandhilfe bemüht. Er fand diese vor allem bei den vielen Gruppen und Komitees, die sich in den USA seit Kriegsende für die Hilfe in Russland engagierten. Dieser Kreis umfasste politisch und gesellschaftlich ein breites Spektrum, das von offenen Sympathisanten der Revolution über Pazifisten bis hin zu landsmannschaftlichen Gruppen und russischen Exilanten reichte. Auf einer Konferenz verschiedener Russlandhilfskomitees im Februar 1921 hatte sich das AFSC als „clearing house“ und Kanal für die Gelder dieser Gruppen nach Russland angeboten. ${ }^{30}$ Thomas selbst war im Juni eine federführende Kraft bei der

28 Cabanes, The Great War and the Origins of Humanitarianism, 1918-1924., 189-247.

29 Im Gegensatz dazu behielten die Sowjets in der Zusammenarbeit mit dem ICRR weitgehend die Kontrolle über die Verteilung der Güter und erhielten deutlich günstigere Konditionen.

30 Wilbur Thomas an Anna Haines 18/2/1921, AFSCA, General Administration, Foreign Service, Country: Russia 1921 (letters from Philadelphia to Russia). 
Gründung des All American Fund for Russian Famine Relief, einer breiten, vor allem von gewerkschaftlichen Gruppen getragenen Hilfskoalition in New York, die prominente Progressive wie Jane Addams zu ihren Unterstützern zählte. ${ }^{31}$ Daneben existierte die All American Commission for Russian Famine Relief, eine von Helen Todd geleitete, hauptsächlich gewerkschaftlich getragene Gruppe. Gewerkschaften wie die Amalgameted Clothing Workers of America waren auch andernorts prominent vertreten und bildeten zu einem späteren Zeitpunkt das Trade Union National Committee for Russian Relief. Es gab zudem das sozialistisch geprägte American Committee for Russian Famine Relief, geleitet von Walter Liggett, David Dubowsky und Paxton Hibben, mit relativ breiter Unterstützung im progressiven Umfeld (unter anderem von Jane Addams und Lilian Wald). Dieses Komitee verteilte seine Gelder anfangs über das (sowjetisch dominierte) Russische Rote Kreuz, ging dann jedoch, auch nach Kritik an dieser zu offensichtlichen Verbindung, dazu über, das AFSC als Kanal zu nutzen. Desweitern existierte der eher moderat linke Russian Famine Fund, unterstützt vom demokratischen Gouverneur New Yorks Al Smith. Dieser setzte sich zwar für die Anerkennung der Sowjetregierung ein, ohne jedoch offen dessen Seite zu ergreifen: Er nutzte das AFSC als Verteiler, weil er die Quäker für „the most thoroughly nonpolitical and independent of the agencies“ hielt, die in Russland aktiv waren. ${ }^{32}$

Dieses Netzwerk, das den Löwenanteil der Spenden für die AFSC-Arbeit in Russland aufbrachte, erwies sich umgekehrt für eine Zusammenarbeit mit Hoover als Bürde. Was diese Gruppen nämlich vereinte, war ein tief empfundenes Misstrauen gegen Hoover und die ARA, deren kaum verhüllte Versuche, „food as a weapon“ gegen die Bolschewiki $\mathrm{zu}$ verwenden, scharf verurteilt wurden. ${ }^{33}$ In diesen Kreisen galt es als Auszeichnung, frei von Hoovers Einfluss in Russland zu arbeiten. Als Wilbur Thomas Anfang des Jahres 1921 in Kontakt mit diesen Gruppen trat, verwendete er die Unabhängigkeit des AFSC bewusst als Eintrittsbillett. „The American Friends Service work in Soviet Russia is entirely independent of the American Relief Administration“, schrieb er etwa an Kenneth Durant, ein Journalist aus Philadelphia und einer der führenden Köpfe hinter den

31 Wilbur Thomas an Anna Haines 6/8/1921, in: AFSCA, General Administration, Foreign Service, Country: Russia 1921 (letters from Philadelphia to Russia).

32 Das verwirrende Netz der Kontakte des AFSC in dieses Milieu spiegelt sich in der Korrespondenz von Thomas und Henry Cadbury (des Vorsitzenden des Russian Committee) wider: AFSCA, General Administration, Foreign Service, Country: Russia 1921 (Russia General); auch die Korrespondenz mit Jane Addams, die mit einigen der Komittees verbunden war gibt Aufschlüsse: AFSCA, General Administration, Foreign Service, Country: Russia 1921 (und 1922) (Individuals: Jane Addams). Daneben eine Übersicht bei Curti, American Philanthropy Abroad, 287-288.

33 Curti, American Philanthropy Abroad, 280. 
pro-sowjetischen Unterstützergruppen. ${ }^{34}$ Die bestehende Zusammenarbeit in Polen und Deutschland ebenso wie jene mit dem Roten Kreuz in Russland erfolge unter der Prämisse vollständiger Unabhängigkeit und alleine entlang zweier Ziele: „1. To relieve suffering. 2. To create a better understanding between the peoples of the various nations and thus promote international good will.“35

Die zur Schau getragene Neutralität mochte durchaus den Intentionen von Thomas und seiner Kollegen im AFSC entsprechen. Die Unabhängigkeit von Hoover als selling point allzu sehr in den Vordergrund zu schieben, konnte sich freilich auch als Bumerang erweisen, wie sich im Umfeld des Riga-Abkommens zwischen der ARA und der Sowjetunion zeigen sollte. Deutlich wurde dies spätestens im August, als Hoover seine Vorstellungen eines Kinderspeisungsprogramms in Russland im Rahmen eines Treffens aller potenziell beteiligten Hilfsorganisationen vorstellte. ${ }^{36}$

Die Anwesenden, einschließlich des AFSC, der durch Rufus Jones und Wilbur Thomas vertreten war, erhielten eine klare Ansage: Um der ,greatest tragedy the world has ever seen“ beizukommen, wollte Hoover eine gewaltige Hilfsmaschinerie auf die Beine stellen, unter klaren Prämissen und zu seinen Bedingungen. Hoovers Arrangement sah die maximale Zentralisierung der Hilfe unter der Ägide der ARA vor. Alle wesentlichen Schritte, vom einheitlichen Spendenaufruf bis hin zur Verteilung der Hilfsgüter in Russland, sollten in ihrer Hand bleiben. Hoover forderte insbesondere, dass die Hilfsgüter der ARA nur von Amerikanern verteilt werden durften. Die einzelnen Hilfsorganisationen könnten im Gegenzug zwar ihre organisatorische Einheit und Identität bei der Hilfe bewahren und sollten innerhalb der ihnen zugeteilten Bezirke weitgehende Autonomie behalten. Die ARA wollte jedoch als alleiniger Ansprechpartner für die sowjetischen Behörden auftreten. Unmissverständlich machte Hoover den Anwesenden klar, dass es bei

34 Kenneth Durant war Herausgeber der sowjetfreundlichen Zeitschrift Soviet Russia und Mitglied im All-American Non-Partisan Famine Relief Committee. Durant äußerte vor und nach dem Abschluss des Washingtoner Abkommens zwischen der ARA und den kleineren Hilfsorganisationen Zweifel an der Unabhängigkeit des AFSC, insbesondere was dessen freien Umgang mit der Sowjetregierung betraf.

35 Wilbur Thomas an Kenneth Durant 6/1/1921, in: AFSCA, General Administration, Foreign Service, Country: Russia 1921 (Russia General).

36 Teilnehmende Organisationen neben den Quäkern waren YMCA, YWCA, JDC, Mennonite Central Committee, ARC, Southern Baptist Convention for Russian Relief, Federal Council of Churches of Christ, National Lutheran Council, National Catholic Welfare Council, Student Friendship Fund, Volga Relief Society, American Committee to aid Russian Scientists. Confidential Memorandum of Discussion at Meeting of European Relief Council in Hoover's Office, 24/8/1921, in: AFSCA, Foreign Service Section, Country: Russia 1921 (Russia General). 
dieser Unternehmung nur die Wahl geben würde, mit der ARA zu arbeiten oder außen vor zu bleiben.

Hoover machte auch aus seinen Motiven keinen Hehl. Verantwortlich für die Katastrophe sei ein irreparabel falsches ökonomisches und politisches System, dessen Beseitigung die Voraussetzung für einen dauerhaften Wiederaufbau darstelle. Die Hilfe sollte deshalb in möglichst direkter Weise an die russische Bevölkerung gelangen und die Kontakte und die Zusammenarbeit mit den sowjetischen Behörden sollten auf ein Minimum begrenzt bleiben.

An einer Stelle des Gesprächs brachte Rufus Jones die Frage nach dem bestehenden angloamerikanischen Hilfsprogramm in Russland auf und regte an, die Quäker könnten diese Arbeit unter der Hoover-Organisation weiterführen: „What we should like would be to have a district assigned to us and maintain our identity. “ Darauf entspann sich ein längeres Streitgespräch zwischen Hoover und den Vertretern des AFSC, das die Konfliktpunkte klar ans Licht brachte. Hoover fragte zunächst, ob das AFSC bereit sei, „to distribute through Americans“, sprich: wie in Deutschland die Zusammenarbeit mit den Briten aufzukündigen. Jones merkte an: „(W)e have always cooperated and are now cooperating with English Friends. It would be difficult to break with them.“ Daraufhin vermerkte das Protokoll den folgenden Austausch: „Hoover: The Russian people consider your work now British relief. RMJ (Rufus Jones): I think they consider it Friends relief.“ Wenig später brachte Hoover das Gespräch auf die Zusammenarbeit der Quäker mit den Sowjetbehörden: „H.H.: Your distribution at present is through the Soviet. RMJ: I suppose it is nominally. WKT (Wilbur Thomas): We have complete control. Hoover: It is distributed through two organizations they have set up. "Das Protokoll weiter: „H.H. and WKT disagreed on question of distribution.“ Schließlich kam es auch zu einem Wortwechsel hinsichtlich der Verbindungen des AFSC ins linke Milieu. Wilbur Thomas warf die Frage auf, ob die Quäker bei einer Zusammenarbeit mit der ARA weiterhin die Gelder pro-sowjetischer Gruppen annehmen könnten. Hoover antwortete: „By all means“, um daraufhin zu einer langen Rede anzuheben: „We might as well be frank about this“, sagte er an die gesamte Runde gewandt (Das Protokoll vermerkte an dieser Stelle: „Hoover turning to the rest of Committee“), „there are various groups of Reds raising money for Russia. They are using the Quakers as a cloak. I think the Quakers should take the money. I don't care what color it is, but the question of fathering such propaganda the Friends must determine for themselves.“ Er sei angeklagt worden „of preventing the spread of Bolshevism in Europe in 1919“. Für seinen Teil, zitierte das Protokoll Hoover, „he was proud to be so accused and considered it a compliment“. Alles, was er getan habe, „he would stick to and would be glad to do it again“. Gleich im Anschluss an dieses Bekenntnis fasste Hoover, nun wieder an Jones gerichtet, das Ergebnis des Austauschs aus seiner Sicht zusam- 
men: „Then the American Friends Service Committee will distribute in their own name as Americans, will deal only with the government through Col. Haskell (den designierten Chef der ARA-Mission in Russland, DM), will have our Transport Department distribute the supplies, will make the conditions of the contract with the Soviets your conditions?“ Die Antwort von Rufus Jones lautete: „Yes“. ${ }^{37}$

Erwartungsgemäß schlug dieses Ergebnis hohe Wellen. Noch vor Abschluss eines Abkommens zwischen AFSC und ARA appellierten entsprechend Vertreter der Unterstützer eindringlich an die Quäker, Hoovers Angebot auszuschlagen. Eine Repräsentantin des All American Fund kündigte an, dass ein solches Abkommen unweigerlich zum Absprung eines Großteils der Unterstützer aus dem radikalen und liberalen Milieu führen würde. Umgekehrt werde man alles tun, um eine eigenständige Quäkerhilfsaktion ohne Verrat an deren Prinzipien der Neutralität und Unparteilichkeit weiterhin zu ermöglichen. ${ }^{38}$

Hoovers Angebot führte ebenso zu Auseinandersetzungen innerhalb des AFSC, so auf einer Sitzung Ende August. Hier traten auch politische Gegensätze zu Tage, die in Deutschland kaum zum Tragen gekommen waren. Während die Appelle linker Gruppen, sich dem Zugriff Hoovers zu entziehen, bei Wilbur Thomas und einer Reihe von weiteren Mitgliedern durchaus auf offene Ohren stießen, blieb Rufus Jones mit einigen konservativeren Friends reserviert. Seiner Ansicht nach würde die breite Masse der Quäker eine offene Abkehr von Hoover zugunsten eines Bündnisses mit radikalen Gruppen nicht akzeptieren.

Politische Gründe allein gaben, zumindest vordergründig, nicht den Ausschlag dafür, dass die Zusammenarbeit mit Hoover kontrovers diskutiert wurde. ${ }^{39}$ Vor allem drei von Hoover gestellte Bedingungen stellten das AFSC vor Probleme: Die Vorgabe, die Hilfsaktion unter rein US-amerikanischen Vorzeichen durchzuführen, implizierte ja erstens wie schon in Deutschland die Auflösung der existierenden amerikanisch-britischen Hilfsmission vor Ort. Zweitens bedeutete der Anspruch der ARA, alle Beziehungen zu den Behörden in Russland zu monopolisieren, dass die Quäker aufgefordert waren, alle seit 1917 etablierten Kontakte auf Eis zu legen. Das widersprach dem Charakter der Quäkermission in Russland, in der „message work“ im Vergleich zu der Mission in Deutschland weitaus höheres Gewicht hatte. Schließlich stellte drittens auch die geforderte Eingliederung

37 Confidential Memorandum of Discussion at Meeting of European Relief Council in Hoover's Office, 24/8/1921, in: AFSCA, Foreign Service Section, Country: Russia 1921 (Russia General).

38 Das Schreiben von Helen Todd (All American Commission for Russian Famine Relief) von 27/ 8/1921 war Teil eines Memorandums für das Executive Board des AFSC 30/8/1921, in: AFSCA, AFSC Minutes 1921 (Executive Board).

39 Memorandums für das Executive Board des AFSC 30/8/1921, in: AFSCA, AFSC Minutes 1921 (Executive Board). 
des AFSC in einen zentralen, von der ARA geleiteten Spendenaufruf die Identität einer Quäkerhilfsmission in Frage. Der vormalige Generalsekretär Tim Nicholson mahnte im August 1921 unmittelbar vor Unterzeichnung des Abkommens mit Hoover an, das AFSC müsse seinen „standard of true relief“ aufrechterhalten. Es gelte die große Stärke der Quäker zu erhalten, die in ,distributing and appealing in this country for relief divorced from political flavor“ liege. Einige weitere Teilnehmer der Debatte lehnten es entschieden ab, als Hoovers „moralisches Feigenblatt“ zu dienen. Andere sprachen sich dagegen dafür aus, die Frage der Identität nicht zu hoch zu bewerten. Ein Teilnehmer monierte, dass ,even though we feel we give a message with our food we ought not to deadlock the relief“. In anderen Worten: Es ging darum, Menschen vor dem Hungertod zu retten. Hierfür sei die Zusammenarbeit mit Hoover, der über ein Vielfaches an Mitteln gebot, ein geringer Preis. ${ }^{40}$

Schließlich setzten sich Ende August nach kontroverser Diskussion diejenigen durch, die mit Rufus Jones für eine Zusammenarbeit mit der ARA plädierten. Dass die Entscheidung des AFSC am Ende erneut für die Zusammenarbeit mit der ARA und gegen eine Fortführung der Hilfsaktion als internationales Quäker-Unternehmen ausfiel, hatte im Wesentlichen zwei Gründe: Zum einen stand das AFSC erneut vor der Wahl, eine relativ kleine Hilfsaktion ohne die ARA durchzuführen oder sich der umfassenderen Mittel Hoovers zu bedienen und eine weitaus größere Wirkung bei der Bekämpfung der Hungersnot zu erzielen. Zum anderen gaben Hoover und seine Mitarbeiter, wie am Anfang des Kapitels gezeigt, recht unverhohlen zu erkennen, dass sie im Stande und im Zweifelsfall auch willens waren, den Quäkern auf ihrem Weg nach Russland Schwierigkeiten zu bereiten. $^{41}$

Somit unterschrieb Rufus Jones Ende August ein Abkommen mit der ARA, das die Auflösung der angloamerikanischen Quäkermission in Russland für den Zeitraum der Aktion bestätigte. Dem AFSC wurde Buzuluk als Einsatzgebiet zugewiesen, was die unmittelbare Weiterführung der Arbeit erleichterte und eine rasche Wiederzusammenführung mit den Briten nach Ende der Aktion erlauben

40 Dass für Hoover nicht allein die überlegenen finanziellen Ressourcen sprachen, wurde in der Diskussion ebenfalls deutlich: Ein weiterer Teilnehmer gab zu bedenken, dass Hoover für die große Mehrzahl der Quäker „a man on a pedestal“ sei. Folglich brauche man „a clear-cut issue before we could take Friends with us in opposing him“. Wieder andere waren der Meinung, man solle es auf eine Konfrontation mit Hoover ankommen lassen, der sich letztlich nicht ins Unrecht setzen und kaum einen Abzug der Quäker erzwingen werde. Das gesamte Protokoll in: Executive Board und Russian Committee joint meeting 23/8/1921, in: AFSCA, AFSC Minutes 1921, hier zitiert nach McFadden and Gorfinkel, Constructive Spirit: Quakers in Revolutionary Russia, 70.

41 Joint meeting Executive board and Russia committee 23/8/1921, in: AFSCA, AFSC Minutes 1921. 
würde. Die ARA stellte dem AFSC im Gegenzug Hilfsgüter für ein Jahr im Wert von 415.000 US-Dollar und zusätzlich den Zugriff auf Transport-, Lager- und Personalkapazitäten der ARA zur Verfügung. Das AFSC verpflichtete sich zur Versorgung einer bestimmten Anzahl von Menschen, zunächst 50.000 Personen. Unmittelbar nach dem Treffen erließ die ARA einen zentralen Spendenaufruf, in dem die Öffentlichkeit angehalten wurde, für Russland bestimmte Spenden nur der ARA und den ihr angeschlossenen Organisationen zukommen zu lassen. ${ }^{42}$

Damit waren die Kontroversen keineswegs beendet. Eher im Gegenteil begann nun für Wilbur Thomas der eigentliche Drahtseilakt, die Entscheidung und ihre Konsequenzen nicht nur den Unterstützern in den USA, sondern vor allem auch den Kollegen in London sowie der angloamerikanischen Hilfsmission in Moskau und Buzuluk zu vermitteln. Anders als in Deutschland handelte es sich bei der russischen Mission um eine lange etablierte und gut bewährte Zusammenarbeit. Gerade die Briten waren es im Frühjahr 1921 gewesen, die sich um eine Erhöhung des amerikanischen Anteils an der Mission gegenüber den zögernden russischen Behörden bemüht hatten. Von der Ankündigung, dass wie in Deutschland eine Trennung bevorstand, wurden sie kalt erwischt. Noch Ende Juli schrieb Ruth Fry an Wilbur Thomas, „Hoover's generous offer“ (den Opfern der russischen Hungersnot nach Maxim Gorkis Appell zu Hilfe zu eilen, DM) werde sicherlich dabei helfen, die Widerstände gegen eine Aufstockung des amerikanischen Personals der Quäkerhilfe zu brechen. ${ }^{43}$ Eine Woche später äußerte Fry sogar die Hoffnung, die Amerikaner würden nun bald in der Lage sein, „to take a larger share in the work as soon as other facilities are given to you“. ${ }^{44}$

Andere waren weniger zuversichtlich: Edith Pye, die in Österreich weilte, äußerte ihr Unbehagen angesichts der Gerüchte, die sich um die Konditionen der ARA-Hilfe rankten, und drückte Sorge über die Zukunft der angloamerikanischen Quäkermission aus, die alleine die Gewähr liefere, dass „purely humanitarian interests shall be represented in Russia“. ${ }^{45}$

42 ARA an AFSC 1/11/1921, in: AFSCA, AFSC Minutes 1921.

43 Ruth Fry vermerkte hierzu, man hoffe, die russische Regierung werde „the claims of the million children whom Hoover offered to feed" höher bewerten als die wenigen verbliebenen amerikanischen Kriegsgefangenen, deren Freilassung Hoover erneut zur Bedingung der Hilfe gemacht hatte. Ruth Fry an Wilbur Thomas 27/7/1921, in: AFSCA, Foreign Service, Country: Russia 1921.

44 Ruth Fry an Wilbur Thomas: 4/8/1921, in: AFSCA, General Administration, Foreign Service, Country: Russia 1921 (letters England) .

45 Edith Pye (Wien) an Ruth Fry 12/8/1921, in: LRSF, Box 7, Panel 5 Folder 5: Russia, Mennonites, Jewish Distribution Committee, Women's International League 1921. 
Nach ihrer Rückkehr aus Genf, wo sie der Gründung der Nansen-Kommission beigewohnt hatte, schrieb Ruth Fry ihrerseits an Wilbur Thomas, offenbar immer noch in der festen Annahme, der Ausbau der gemeinsamen Quäkermission genieße für beide Seiten nach wie vor oberste Priorität. Den Wert einer möglichen Zusammenarbeit mit der Nansen-Organisation bemaß sie danach, ob dieser den unabhängigen Charakter der gemeinsamen Quäkerhilfe unangetastet lasse - ein weiteres Indiz dafür, dass Fry keinen Gedanken daran verwendete, das AFSC könne diese Zusammenarbeit in Frage stellen. ${ }^{46}$

Zum selben Zeitpunkt versuchte Wilbur Thomas, den beiden Leitern der russischen Hilfsmission, Arthur Watts und Anna Haines, behutsam die Nöte zu schildern, denen das AFSC im Umgang mit Hoovers Angebot ausgesetzt war. Man blicke, so Thomas, gegenwärtig mit einer Mischung aus Sorge und Hoffnung auf die Verhandlungen, die Hoover mit den Bolschewiki führe, hieß es in seinem Schreiben. Scheiterten diese Verhandlungen, so würden sie die Hilfe der Quäker keinesfalls unbeschädigt lassen, „for if they fall through they will turn the tide of public opinion against Russian relief in a very large way“ und es werde auch für die Quäker schwer, Unterstützung von Großspendern zu bekommen. Dies sei womöglich finanziell durch die Unterstützung der Gewerkschaften abzufedern. Die eigentliche politische Gefahr bestehe darin, dass das State Department im Fall des Scheiterns der Verhandlungen sicher Druck auf das AFSC ausüben würde, die russische Hilfe vor Ort ebenfalls einzustellen. Ob das AFSC dann tatsächlich gegen den ausdrücklichen Wunsch der Regierung in Russland weiterarbeiten werde, sei fraglich. ${ }^{47}$ Rufus Jones erläuterte kurze Zeit später, das AFSC könne sich der Tatsache nicht verschließen, dass die ARA ein Vielfaches der Ressourcen bieten könne, als den Quäkern durch die Unterstützung der Gewerkschaften und anderer Gruppen in Aussicht stünde. Jones berichtete auch von einem Gespräch mit dem Leiter der ARA, William Haskell, in dem dieser sich offen für eine Lösung gezeigt habe, bei der die Zusammenarbeit mit den Briten unter amerikanischer Führung im Wesentlichen weitergeführt werden, oder gar den britischen Quäkern von der ARA ein eigener Bezirk zugewiesen werden könnte. ${ }^{48}$

Erst gegen Ende des Monats, als die Nachrichten über die Details des Abkommens des AFSC mit der ARA durchzusickern begannen, veränderte sich der Ton auf britischer Seite. Ruth Fry versicherte Thomas ihres „warm sense of

46 Ruth Fry an Wilbur Thomas 18/8/1921, in: AFSCA, General Administration, Foreign Service, Country: Russia 1921 (letters to/from England).

47 Wilbur Thomas an Anna Haines/Arthur Watts 18/8/1921, in: AFSCA, General Administration, Foreign Service, Country: Russia 1921 (letters from Philadelphia to Russia).

48 Rufus Jones an Ruth Fry 24/8/1921, AFSCA, General Administration, Foreign Service, Country: Russia 1921 (letters England and Russia) 
sympathy“, insistierte jedoch gleichzeitig, dass die gemeinsame Sache nicht geopfert werden dürfe: „We know that it may be a choice between a comparatively small work on Friends lines or a much larger one in co-operation with Hoover, but we feel that the value of our work is not measured purely by its size.“

Die finanziellen Nachteile könnten durch eine engere Zusammenarbeit mit der Nansen-Aktion aufgewogen werden, die zudem „greater elasticity“ biete, , as it is not national work but an international one“ . ${ }^{49}$ Ein fast zeitgleich abgeschicktes Telegramm wurde in der Sache noch deutlicher: „Advise freedom from Hoover and acting internationally. (...) English Friends strong desire co-operate with you in new work famine area which Hoover suggestion precludes. Consider Friends direct dealings with Soviet of greatest value (...). “50 Fry konnte allerdings das finanzielle Argument nicht gänzlich entkräften, weil die Erfolgsaussichten Nansens zu diesem Zeitpunkt mehr als fragwürdig erschienen. ${ }^{51}$

Mitte September teilte Thomas die endgültige Entscheidung des AFSC mit, mit Hoover zu arbeiten und folglich einer Auflösung der gemeinsamen Mission zuzustimmen. Fry gegenüber betonte er, es handele sich um „clothes we have to wear for the time being in order to give our message“, ohne dass dies etwas an der ideellen Einheit der Quäker ändere. „One at heart and purpose in all our work“, bleibe man auch in der Zukunft. Man hoffe zudem, zumindest lose kooperieren zu können und bemühe sich um die Zuteilung benachbarter Distrikte. ${ }^{52}$ Fry zeigte sich tief enttäuscht, bemühte sich jedoch darum, einen konzilianten Ton zu bewahren und auf die Möglichkeit einer nahtlosen Wiederaufnahme der Zusammenarbeit hinzuweisen, sobald sich die Umstände änderten. ${ }^{53}$

Dagegen fiel die Reaktion der Führung der Moskauer Quäkermission ungleich schärfer aus. Hier löste die Ankündigung der Auflösung der gemeinsamen Mission ungläubiges Entsetzen und offene Empörung aus. Aus dem Ton, in dem Wilbur Thomas Arthur Watts und Anna Haines die Entscheidung mitteilte, wird deutlich, dass er sich über die zu erwartenden Reaktionen vollauf im Klaren war: „This will

49 Ruth Fry an Wilbur Thomas 31/8/1921, in: AFSCA, General Administration, Foreign Service, Country: Russia 1921 (letters England and Russia).

50 Ruth Fry an Wilbur Thomas 29/8/1921, in: AFSCA, General Administration, Foreign Service, Country: Russia 1921 (letters to/from England).

51 An diesen Erfolg war jedoch auch der eines künftigen britischen National Appeal geknüpft, der als mögliche alternative Geldquelle für die Quäkerhilfe zur Verfügung stehen würde. Ruth Fry an Wilbur Thomas 9/9/1921, in: AFSCA, General Administration, Foreign Service, Country: Russia 1921 (letters to/from England).

52 Wilbur Thomas an Ruth Fry 16/9/1921, zitiert nach McFadden and Gorfinkel, Constructive Spirit: Quakers in Revolutionary Russia, 67.

53 Ruth Fry an Wilbur Thomas 5/10/1921, in: AFSCA, AFSCA, General Administration, Foreign Service, Country: Russia 1921 (letters to/from England). 
cause you a considerable amount of anxiety and perhaps you will feel like criticizing us very severely. “54 In der Tat waren weder Haines noch Watts, die beide gemeinsam seit langer Zeit in Russland tätig waren, gute Kontakte zu den Sowjetbehörden pflegten und der Revolution ein gutes Maß an Sympathien entgegenbrachten, bereit, die Entscheidung ohne Weiteres zu akzeptieren. Beide waren bekanntermaßen entschiedene Gegner einer engen Bindung an die ARA. Eine unabhängige angloamerikanische Quäkermission sahen sie als beste Gewähr gegen eine Vereinnahmung durch „politische“ Interessen, wie sie Hoover aus ihrer Sicht repräsentierte.

Haines antwortete Thomas, indem sie nochmals die Punkte zusammenfasste, die aus ihrer Sicht gegen eine Zusammenarbeit mit Hoover sprachen: Nicht nur sei die ARA in Russland extrem unpopulär, ihre Ziele unterschieden sich diametral von denen der Quäker: Das Konzept der Quäker für Russland sei langfristig, das der ARA kurzfristig angelegt. Auf lange Sicht, so argumentierte sie, sei der kaum vermeidbare Verlust des bisherigen Unterstützermilieus in den USA nicht zu kompensieren. All diejenigen, welche die Quäker bislang als frei von „high politics and high finance“ gesehen hätten, seien unwiederbringlich verloren. Wenig realistisch betrachtete sie die Aussichten, die radikalen und linken Kräfte dazu zu bewegen, ihr Geld an die britischen Quäker weiterzuleiten. Schließlich warf Haines ein, ein Schritt von solcher Tragweite, wie es das Abkommen mit Hoover darstelle, könne unmöglich ohne vorherige Absprache mit der „unit in the field“ erfolgen. ${ }^{55}$ Für Arthur Watts war die Frage der Einheit der angloamerikanischen Quäkermission eine noch viel elementarere. Er war durch den Auszug der Amerikaner nicht nur vor die Aufgabe gestellt, eine britische Hilfsmission ohne Mittel zu etablieren. Er sah sich darüber hinaus um die Früchte seiner jahrelangen Arbeit gebracht. Für ihn kam der Schritt des AFSC folglich einem Verrat gleich, umso mehr, als er für sich reklamierte, einen großen Anteil daran gehabt zu haben, den Amerikanern, unter anderen Haines und Nancy Babb, gegen die Widerstände der Bolschewiki die Rückkehr nach Russland zu ebnen. „Now you have gone and thrown it all away“, warf er Thomas mit einiger Bitterkeit vor. „DO YOU WONDER THAT I FEEL THAT I HAVE WASTED TWO YEARS AS FAR AS AMERICAN FRIENDS ARE CONCERNED (Kapitalschrift im Original, DM).“ Watts machte Thomas dafür verantwortlich, „to bring to Russia the picture of the Friends International Service becoming two separate organizations one of which at least will be NATIONALISTIC and the other will have a hard job to explain that it is not also of the same

54 Wilbur Thomas an Arthur Watts und Anna Haines 17/9/1921, in: AFSCA, General Administration, Foreign Service, Country: Russia 1921 (letters from Philadelphia to Russia).

55 Anna Haines an Wilbur Thomas 20/09/1921, in: AFSCA, General Administration, Foreign Service, Country: Russia 1921 (letters to Philadelphia from Russia) 
nature but representing another nation“. Es bleibe nur die Hoffnung, schloss Watts, dass die Quäker Hoovers kurzfristiges Engagement überdauern würden, ,and if we are not completely buried under Stars and Stripes or Union Jacks or have not been swallowed by lions and eagles we may try again to re-establish by November 1922 what we have lost in 1921“.56

Bis zum Ende der Mission konnten die Verwerfungen zwischen dem home office in Philadelphia und den Mitgliedern der Hilfsmission in Moskau und Buzuluk nie vollständig gekittet werden. Zwar gab es unter den amerikanischen Mitgliedern der Mission auch vereinzelte Stimmen wie die von Nancy Babb, die eine Zusammenarbeit mit der ARA begrüßte, weil sie sich hiervon eine Änderung der ihrer Auffassung nach zu sowjetfreundlichen Haltung der Mission versprach. So beschwerte sich Babb über Arthur Watts und die britischen Quäker und zeigte sich überzeugt, mit der ARA werde die Aktion ihren politischen Charakter verlieren: „I do not believe we as Friends should be continually knocking any relief organisation now in Russia or in any way refer to any other organization as being political when we at present are so justly accused of being here to help the government more than the people as a whole. ${ }^{\text {"57 }}$ Die Mehrzahl der Helfer vor Ort empfand die Trennung zumindest anfänglich gleichwohl als Verrat an den gemeinsamen Idealen.

\section{Cold and hunger in every eye. Der AFSC-Einsatz 1921-1922}

Dessen ungeachtet agierte das AFSC ab Oktober 1921 als ein Teil der ARA-Aktion. Bertrand Patenaude zeichnet in seiner umfassenden Studie „Big Show in Bololand“ ein monumentales Gemälde des Einsatzes der ARA in Russland. Sie verdeutlicht die gewaltige Maschinerie, welche die Hoover-Organisation in Russland während der letzten Monate des Jahres 1921 in Gang brachte. Es gelang ihr auf dem Höhepunkt der Aktion, täglich bis zu elf Millionen Menschen mit Nahrung, Kleidung und Medizin zu versorgen - in einem Gebiet, das sich in etwa von Kasan im Norden Russlands bis zu den Mündungen von Wolga und Don im Schwarzen

56 Watts verwies auf den „great deal of energy and personal influence being used to secure permission for Nancy Babb as an exception to the standing objection to Americans"; Arthur Watts an Wilbur Thomas 28/10/1921, in: AFSCA, General Administration, Foreign Service, Country: Russia 1921 (letters to Philadelphia from Russia).

57 Nancy Babb an Wilbur Thomas 10/11/1921, in: AFSCA, General Administration, Foreign Service, Country: Russia 1921 (letters to Philadelphia from Russia), zitiert nach McFadden and Gorfinkel, Constructive Spirit: Quakers in Revolutionary Russia, 67. 
und Kaspischen Meer über nahezu tausend Kilometer erstreckte. ${ }^{58}$ Von der bis 1923 in Russland von ausländischen Hilfsorganisationen geleisteten Hilfe entfielen rund 83 Prozent auf die ARA. ${ }^{59}$

Vor diesem Hintergrund war das AFSC-Kontingent nur ein Rad in einem größeren Getriebe. Im Dezember 1921 erreichte die erste Gruppe von frischen amerikanischen Helfern den Bezirk Samara, der nun zwischen den britischen und amerikanischen Missionen aufgeteilt wurde. ${ }^{60}$ Als Leiter des amerikanischen Kontingents diente Murray Kenworthy, ein Quäker-Pastor und Farmer aus dem Mittleren Westen der USA. ${ }^{61}$ Buzuluk blieb für beide Missionen das Zentrum ihrer Hilfsmission, was bedeutet, dass de facto ein kleiner Teil der angloamerikanischen Mission, wenngleich organisatorisch getrennt, weiterbestand. Der Unterschied bestand im Wesentlichen darin, dass die AFSC-Helfer ihre Unterstützung von der ARA bezogen, während die Briten ihre Arbeit vorwiegend über einen zentral geleiteten britischen Spendenaufruf sowie aus den vergleichsweise bescheidenen Zuteilungen des Nansen-Komitees finanzierten. Bis zu einem gewissen Grad wurden dadurch neue Realitäten geschaffen. Caroline Norment, die ab Frühjahr 1922 das AFSC-Kontingent in Moskau verstärkte, hielt eine baldige Wiederzusammenlegung der beiden Teilmissionen für wenig ratsam - die britische Hilfsoperation schien ihr im Vergleich zu den AFSC-Helfern unorganisiert und demoralisiert, wofür nicht zuletzt die fehlenden Ressourcen verantwortlich zeichneten. ${ }^{62}$

Wie das Verhältnis zur ARA sich konkret gestaltete, dazu ergibt sich anhand der Quellen ein wenig einheitliches Bild. Patenaude betont vor allem die Zwistigkeiten, die ihre Ursache vor allem in der nach Auffassung der ARA ineffizienten Arbeitsweise der Quäker und deren „softer brand of relief“ gehabt habe. ${ }^{63}$ Andere Quellen vermitteln das Bild eines (auch geografisch) distanzierten, aber im Wesentlichen reibungsarmen Verhältnisses. Das AFSC lieferte der ARA in Moskau

58 Patenaude, The Big Show in Bololand: The American Relief Expedition to Soviet Russia in the Famine of 1921.

59 Zum Vergleich: In etwa 13 Prozent entfielen auf alle unter dem Dach des International Committee for the Relief of Russia (ICRR) agierenden Organisationen. Vogt, „Fridtjof Nansen and European Food Aid to Russia and the Ukraine 1921-1923,“ 41.

60 Einen Überblick über die Arbeit bietet AFSC Annual Report (1922), 7-9.

61 Kenworthys in vieler Hinsicht repräsentative Geschichte eines der ersten relief worker des AFSC zu dieser Zeit findet sich in einer von seinem Sohn verfassten Biografie: Leonard Kenworthy, Living in a Larger World: The Life of Murray S. Kenworthy (Richmond: Friends United Press, 1986). 62 „Inadvisable“ sei eine Wiederzusammenlegung, meinte hierzu Caroline Norment. Caroline Norment an Wilbur Thomas 26/6/1922, in: AFSCA, General Administration, Foreign Service, Country: Russia 1922 (correspondence to Philadelphia from Russia).

63 Siehe vor allem Patenaude, Big Show, 139-142; hier zitiert 141. 
Berichte nach den von der Hoover-Organisation vorgegebenen Standards und erhielt die Hilfsgüter über das ARA-Verteilungssystem. Die Vereinbarungen, die zur Zusammenarbeit zwischen Kenworthy und dem Leiter der ARA-Operation William Haskell zu Anfang getroffen worden waren, wurden von Quäkerseite als lose empfunden. Caroline Norment in Moskau fasste die vorherrschende Ansicht unter den Mitgliedern des amerikanischen Kontingents nach einem guten halben Jahr der Arbeit unter dem Dach der ARA folgendermaßen zusammen: ,We do not consider ourselves an integral working part of the ARA organization and do not carry on our relief work in conformity with their plan and program.“64

Auch inwieweit die offizielle Trennung von den britischen Quäkern tatsächlich eine Änderung in der Politik gegenüber den lokalen und nationalen Behörden mit sich brachte (so wie es das Abkommen mit der ARA vorsah), ist im Einzelnen schwer nachzuvollziehen. Über ein offizielles Abkommen, wie es das AFSC in Deutschland mit der Regierung und dem DZA unterzeichnet hatte, verfügten die Quäker in Russland nicht, weder vor noch während der ARA-Operation. Insofern war das Verhältnis von Kontinuität und Bruch schwer zu bemessen. ${ }^{65}$ Aus den Berichten der Leitung vor Ort lässt sich lediglich schließen, dass diese Kontakte sowohl in Moskau als auch in Buzuluk weiterbestanden. Wieviel davon informell geschah und inwieweit die ARA in die Beziehungen tatsächlich direkt eingriff darüber lassen die vorhandenen Quellen keine endgültigen Schlüsse zu. Nach allen verfügbaren Berichten war die Zusammenarbeit mit lokalen Vertretern der Sowjetmacht sachlich. Dass die Regierung umgekehrt durchaus willens war, ein Auge auf die Kontakte der Quäker vor Ort zu werfen, wurde im Fall von Nancy Babb deutlich. Sie griff auf ihrem relativ isolierten Posten außerhalb von Buzuluk auf lange etablierte Netzwerke mit - vielmals den Bolschewiki gegenüber oppositionell eingestellten - Vertretern der lokalen Intelligenz zurück, was ein ums andere Mal zu Schwierigkeiten führte. ${ }^{66}$

Für alle Beteiligten des Einsatzes war die Hilfsaktion ein einschneidendes Erlebnis, das auch für diejenigen, die Erfahrungen aus dem Krieg oder dem Einsatz in Deutschland mitgebracht hatten, alles in den Schatten stellte. Bei allem

64 Caroline Norment an Wilbur Thomas 23/9/1922, in: AFSCA, General Administration, Foreign Service, Country: Russia 1922 (correspondence to Philadelphia from Russia).

65 „Our position here is in many ways unnecessarily complicated by the fact that we have no very definite binding agreement with the Central Government or its representatives such as we had for instance in Germany with the DZA and such as both the ARA and Nansen organization have here." Caroline Norment an Wilbur Thomas 14/8/1922, in: AFSCA, General Administration, Foreign Service, Country: Russia 1922 (correspondence to Philadelphia from Russia).

66 Murray Kenworthy an Wilbur Thomas, (wahrscheinlich April) 1922, in: AFSCA, General Administration, Foreign Service, Country: Russia 1922 (correspondence to Philadelphia from Russia). 
Aufwand und den gewaltigen Mengen an Hilfsgütern, welche die Hilfsorganisationen ins Land brachten, waren die Opferzahlen immer noch enorm. Im Distrikt Samara, der besonders hart von der Not betroffen war, kam in manchen Bezirken im ersten Hungerwinter nach Schätzungen der Quäker vor Ort rund ein Drittel der erwachsenen Bevölkerung durch die Folgen des Hungers sowie durch Seuchen ums Leben. Besonders hoch war zudem die Kindersterblichkeit. Die Speisungsaktion des AFSC war vor diesem Hintergrund trotz der immensen Reichweite nach Ansicht vieler vor Ort nicht mehr als der Tropfen auf dem heißen Stein. ${ }^{67}$

Der Horror der Hungersnot wird in den Briefen und Berichten vieler Quäkerhelferinnen und -helfer greifbar: Anna Haines schrieb in einem der ersten Berichte aus den Hungergebieten vom Grauen überfüllter Waisenhäuser, die täglich riesige Zahlen von neu ankommenden Kindern zu verzeichnen hatten, deren Eltern verhungert waren. ${ }^{68}$ Nancy Babb berichtete von den verstörenden Bildern und Geschichten, mit denen die Mission täglich konfrontiert war, von „numerous cases of suicide, mothers killing and eating their own children, people subsisting on the corpses which have been delayed burial". ${ }^{69}$ Überall wohin man blickte, war nichts als Verzweiflung und Hoffnungslosigkeit, vor der Worte versagten, oder wie ein anderer Helfer anmerkte: „There is cold and hunger in every eye and heart. “70

Auch unter den Quäkern selbst forderte der Einsatz einen hohen Preis. Mehrere Mitglieder der Delegation, darunter Murray Kenworthy, Nancy Babb und Beulah Hurley, erkrankten selbst an Typhus, der, wie Babb anmerkte, „prevalent in every home and on every train“ war. ${ }^{71}$ Im Frühjahr 1922, wurde die Situation so prekär, dass Kenworthy Philadelphia dringlich um eine Aufstockung des Personals mit „people physically fit“ bat, als „reserve force“ für die geschwächte Einheit. $^{72}$ Nicht geringer als die körperlichen waren die mentalen Belastungen, nicht

67 Laut Jahresbericht für das Jahr 1921 versorgten die amerikanischen Quäker im September diesen Jahres rund 150.000 Erwachsene und Kinder mit Nahrung. Dafür standen ihnen Mais aus den Zuteilungen der ARA im Wert von rund 25.000 US-Dollar zur Verfügung. AFSC Annual Report (1922), 9.

68 Anna Haines an Wilbur Thomas 27/11/1921, in: AFSCA, General Administration, Foreign Service, Country: Russia 1921 (fund raising letters).

69 Nancy Babb an Wilbur Thomas 8/3/1922, in: AFSCA, General Administration, Foreign Service, Country: Russia 1922 (correspondence to Philadelphia from Russia).

70 N.N. member of AFSC unit an Wilbur Thomas 25/4/1922, in: AFSCA, General Administration, Foreign Service, Country: Russia 1922 (correspondence to Philadelphia from Russia).

71 Nancy Babb an Wilbur Thomas 8/3/1922, in: AFSCA, Foreign Service Section, Country: Russia 1922 (correspondence to Philadelphia from Russia) .

72 Murray Kenworthy an Wilbur Thomas 17/3/1922, in: AFSCA, Foreign Service Section, Country: Russia 1922. 
zuletzt da Informationen aus der Außenwelt nur mit beträchtlicher Verzögerung in den Hungergebieten eintrafen und ein Gefühl der Isolation erzeugten. ${ }^{73}$

Erst im Frühsommer 1922 begann sich die Lage in den Hungergebieten allmählich zu bessern. Wenngleich die Ernte dieses Jahres gut war, gab es auch im zweiten Winter noch unzählige Hungertote. Zuvor hatte die ARA im Juli bereits das Ende ihrer Hilfsaktion angekündigt und tatsächlich verließen die letzten ARARepräsentanten im September 1922 Russland..$^{74}$

\section{Im Namen des JDC. Die Quäker in Belarus}

Unter gänzlich anderen Vorzeichen verlief ein Strang der Hungerhilfe, bei dem das AFSC parallel zu seiner Beteiligung an der ARA-Hilfsaktion eine Zusammenarbeit mit dem American Joint Jewish Distribution Committee (JDC) einging. Wie das AFSC war das bereits zu Kriegsanfang 1914 gegründete JDC ein Ergebnis der humanitären Mobilisierungswelle, die der Weltkrieg in den USA ausgelöst hatte. Es diente dabei als Klammer älterer jüdisch-amerikanischer Hilfsinitiativen, die sich insbesondere der vielfach Verfolgungen ausgesetzten jüdischen Bevölkerung Ostund Südosteuropas sowie jüdischen Einwanderern nach den USA annahmen. ${ }^{75}$ Mit dem AFSC hatte es erste Berührungspunkte bereits während der Aktion in Deutschland gegeben, als das JDC als assoziiertes Mitglied der Hoover-Organisation Gelder an das AFSC weiterleitete. Darüber hinaus bestanden Kontakte zwischen Vertretern des JDC und des AFSC auf persönlicher Ebene, die sich im Rahmen der russischen Aktion nutzen ließen. Beide Organisationen gehörten zu der Gruppe der Hilfsagenturen, die im Sommer 1921 zum Teil der ARA-Operation in Russland wurden. Daneben verfolgte das JDC jedoch auch eigene Interessen und operierte in Osteuropa auf Basis eigenständiger Strukturen. ${ }^{76}$

Bereits zuvor, Anfang 1921, war Herbert Lehman ${ }^{77}$ als Vertreter des JDC an das AFSC mit dem Vorschlag herangetreten, im Auftrag des JDC Hungerhilfe in einigen

73 So erfuhr Murray Kenworthy von der schweren Erkrankung und dem Tod seiner Frau in Ohio erst Tage nach dem Ereignis über das zentrale Nachrichtensystem der ARA. Wilbur Thomas an AFSC Board 24/5/1922, in: AFSCA, Foreign Service Section, Country: Russia 1922.

74 AFSC Annual Report 1923, 11-14.

75 Ein hervorragendes Panorama der Aktivitäten des JDC in dieser frühen Phase bietet die jüngst erschienene Studie von Jaclyn Granick, International Jewish Humanitarianism in the Age of the Great War (Cambridge: Cambridge University Press, 2021).

76 Zur Arbeit des JDC in der unmittelbaren Nachkriegszeit in knapper Form auch Curti, American Philanthropy Abroad, 293-300.

77 Herbert Lehman (1878-1963) war 1933 bis 1942 Gouverneur von New York und von 1943 bis 1946 Direktor der United Nations Relief and Rehabilitation Administration (UNRRA). 
Bezirken mit einem hohen jüdischen Bevölkerungsanteil in Weißrussland und der Ukraine zu leisten. ${ }^{78}$ Aus Sicht des JDC hatte eine solche Regelung, bei der die Quäker als Stellvertreter jüdischer Hilfsorganisationen fungieren würden, eine Reihe von Vorteilen. Insbesondere die weitverbreiteten anti-jüdischen Ressentiments im Einsatzgebiet legten einen solchen Schritt nahe. Dies beruhte laut Lehman auf der schmerzvollen Erfahrung, „that if the work is done by Jews for Jews that it fosters the anti-semitic feeling“ “. ${ }^{79}$ In den USA sähen sich jüdische Organisationen gleichzeitig dem Generalverdacht ausgesetzt, mit den Bolschewiki zu sympathisieren. Hier kamen die Quäker ins Spiel, da ,the public does not suspect Friends of political sympathies““ ${ }^{80}$ Wie Thomas wenig später gegenüber Anna Haines erläuterte, hatte sich das JDC aus diesem Grund entschieden, „to submerge its identity in Russian relief and ask the Friends to carry it on, feeling that they will be relieving misery just as much and confident that it will be done as honestly and efficiently as their organization could do it“". ${ }^{1}$

Bevor ein solches Abkommen in Kraft treten konnte, mussten zunächst jedoch noch Hürden beseitigt werden. Der ursprüngliche Wunsch des JDC, die Hilfe vor allem der jüdischen Bevölkerung zukommen zu lassen, wurde von Seiten des AFSC als „stumbling block“ gesehen. Aus Sicht der Quäker sollte der Bedarf an Hilfe unabhängig von religiöser Zugehörigkeit das einzig gültige Kriterium sein. ${ }^{82}$ Auch beanspruchte das AFSC die Leitung des Unternehmens für sich; jüdisches Personal vor Ort sollte in die Hilfsmissionen eingegliedert werden, jedoch nicht als offizielle Vertretung des JDC firmieren. ${ }^{83}$ In der Korrespondenz zwischen dem AFSC in Philadelphia und der Mission in Moskau wurden weitergehende Vorbe-

78 Die gesamten Verhandlungen zwischen JDC und AFSC sind ausführlich dokumentiert in einem Memorandum on the Future Work of the Friends International Service in the Russian Socialist Federated Republic 18/8/1921, in: AFSCA, Foreign Service, Country: Russia 1921 (Russia General).

79 Wilbur Thomas an Anna Haines 1/4/1921, in: AFSCA, Foreign Service, Country: Russia 1921 (letters from Philadelphia to Russia).

80 Gespräch Lehman und Wilbur Thomas im April 1921 und die weiteren Verhandlungen, wiedergegeben im Brief von James Norton (Stellvertretender Executive Secretary AFSC) an Anna Haines 15/4/1921, in: AFSCA, Foreign Service, Country: Russia 1921 (letters from Philadelphia to Russia).

81 James Norton (Stellvertretender Executive Secretary AFSC) an Anna Haines 15/4/1921, in: AFSCA, Foreign Service, Country: Russia 1921 (letters from Philadelphia to Russia).

82 James Norton (AFSC) an Anna Haines 19/4/1921, in: AFSCA, Foreign Service, Country: Russia 1921 (letters from Philadelphia to Russia).

83 Das New York Committee des JDC hatte sich gegen das Abkommen zwischen AFSC und JDC gestellt und darauf bestanden, vier jüdische Repräsentanten in die Aktion zu integrieren. Wilbur Thomas an Arthur Watts 20/6/1921, in: AFSCA, Foreign Service, Country: Russia 1921 (letters from Philadelphia to Russia). 
halte deutlich: Unter den Quäkern vor Ort herrschte „some hesitation in associating Jews in the actual management in Russia“. Das allgemeine Gefühl sei, „that if they (das JDC, DM) entrust us at all with their work, they will have to trust us entirely, reserving to themselves the right to discontinue the arrangement if they find in practical working, the confidence they have reposed in us, has been misplaced“. ${ }^{4}$

Schließlich schlossen AFSC und JDC im Juni 1921 ein Abkommen, das den Vorgaben des AFSC weitgehend entsprach: Die Quäker würden in Gebieten mit großem jüdischem Bevölkerungsanteil aktiv werden, dabei jedoch Juden und Nicht-Juden gleichermaßen in die Hilfe einbeziehen. Die Quäker würden die gesamte Verteilung der Güter übernehmen und damit auch das Gesicht der Aktion darstellen, das JDC dagegen würde nur einige wenige eigene Helfer zur Unterstützung und als Signal an die eigenen Spender entsenden. ${ }^{85}$

Im August und im Angesicht der heraufziehenden Hungersnot begannen die Verhandlungen zwischen Vertretern des JDC und Haines und Watts in Moskau, wobei von Anfang an klar war, dass die Hilfsmission sich aus britischen und amerikanischen Quäkern zusammensetzen würde. Strittiger war das Einsatzgebiet. Aus Sicht des JDC waren Minsk und Kiew, wo der Verfolgungsdruck auf die jüdischen Gemeinden besonders stark war, am besten für einen Einsatz geeignet. Die Quäker waren skeptisch, vor allem weil, wie Anna Haines einwandte, ein solcher Einsatz ,would create ill feeling here if too great a proportion of our help were sent exclusively to Jewish districts“. Eine bessere Lösung war aus ihrer Sicht die Verteilung der JDC-Hilfsgüter in Katarinoslaw und Samara, im Kerngebiet der Quäkerhilfe, wo es viele jüdische Flüchtlinge gab. Als das JDC jedoch auf Belarus als primärem Einsatzgebiet bestand, einigte man sich darauf, eine Gruppe unter der Leitung des britischen Quäkers William Albright nach Minsk zu entsenden. ${ }^{86}$

An den Grundlagen dieser Arbeit änderte sich für die folgenden rund zwei Jahre kaum etwas, auch nicht als beide Organisationen sich an der ARA-Hilfsoperation beteiligten. Während das JDC Hoover etwa zwei Millionen US-Dollar für die Hilfe in der Wolga-Region zur Verfügung stellte und das AFSC seine Helfer nach Buzuluk entsandte, arbeitete eine vorwiegend britische Gruppe von Quäkern bis 1923 in Minsk mit Geld des JDC. Gelegentliche Kritik daran, dass die Quäker

84 William Albright an James Norton (AFSC), 28/1/1922, in: AFSCA, Foreign Service, Country: Russia 1921 (JDC). Aus der Sicht des JDC siehe Granick, International Jewish Humanitarianism, 99-100.

85 Ruth Fry an Wilbur Thomas 11/8/1921, in: AFSCA, Foreign Service, Country: Russia 1921 (Letters England - Phila.)

86 Anna Haines an Wilbur Thomas 12/8/1921, in: AFSCA, Foreign Service, Country: Russia 1921 (letters to Philadelphia from Russia). 
Ressourcen in einem Gebiet einsetzten, das nicht $\mathrm{zu}$ den Brennpunkten der Hungersnot zählte (seitens der Quäkermission in Moskau), am „internationalen“ Charakter der Mission (seitens der ARA) oder an der Weigerung der Hilfsmission, jüdisch-amerikanische Gelder gezielt zur Behebung jüdischen Leidens zu beheben (seitens des JDC) änderte nichts daran, dass dieser Aspekt der Hilfsarbeit zu den vergleichsweise reibungsfreien Bereichen der Hilfe zählte. Darüber hinaus kann der Einsatz auch als Indikator für die wachsende Anerkennung gelten, die das AFSC als Hilfsorganisation erfuhr, und insbesondere für den Erfolg der Selbstdarstellung als eine besonders neutrale, rein humanitären Kriterien verpflichtete Kraft. Gleichzeitig warf er ein Schlaglicht auf die Bandbreite amerikanischer Hilfstätigkeit, in der die Quäker sich zwischen der „flaggenschwenkenden“ Hilfe der ARA und dem JDC positionierten, das nolens volens versuchte, bei seinen Aktivitäten möglichst unsichtbar zu bleiben.

\section{An amazing campaign. Die Debatte um den Einsatz in der Sowjetunion geht weiter}

Indes setzte sich in den USA der Konflikt zwischen Hoover und seinen Gegnern unvermittelt fort und ließ das AFSC ein ums andere Mal zwischen die Stühle geraten. Anders als anfänglich erwartet, zeitigte die Allianz der Quäker mit der ARA keinen unmittelbaren Bruch mit dem linken und liberalen Unterstützermilieu. Bei aller Abneigung gegen Hoover zeigten sich viele dieser Gruppen, auch aufgrund fehlender Alternativen, weiterhin bereit, ihre Spendengelder über das AFSC in die Sowjetunion fließen zu lassen. Den landesweiten Appell Hoovers, Geld für Russland fortan nur noch über die ARA und ihr angeschlossene Organisationen laufen zu lassen, fassten sie gleichwohl als die Kampfansage auf, als die sie ja in der Tat gemeint war. Im Gegenzug ließen sie keine Gelegenheit ungenutzt, um Hoovers Rolle in ein schlechtes Licht zu rücken. Für den AFSC besonders heikel war, dass die linke und liberale Presse dabei immer wieder den Umgang der ARA mit den Quäkerorganisationen in Russland als Beweis für Hoovers primär politische Zielsetzungen ins Feld führte. Die russische Regierung tat ein Übriges, einen Keil zwischen die amerikanischen Hilfsorganisationen zu treiben, indem sie den vermeintlichen Kontrast zwischen der „neutralen“ Art und Weise, wie die Quäker Hilfe zu leisten bereit waren, und den „politischen“ Motiven der ARA herausstrich. Diese Umstände erklären zu einem guten Teil die zunehmende Gereiztheit der ARA-Offiziellen im Umgang mit dem AFSC, wobei implizit der Vorwurf der Illoyalität gegenüber den von der ARA vertretenen amerikanischen Interessen mitschwang. 
Diese kritische Haltung der ARA brachte Wilbur Thomas bereits im September 1921, nur einen Monat nach Abschluss des Vertrags mit der ARA, dazu, den Rückzug des AFSC aus Russland zu erwägen und darüber mit Rufus Jones zu beraten. Thomas regte an, alles in den USA gesammelte Geld könnte an die britischen Quäker weitergeleitet werden, während die Arbeit für das JDC in der Ukraine und Belarus weiterlaufen könnte wie bisher. ${ }^{87}$ Jones versuchte daraufhin im Gespräch mit Hoover eine für alle Seiten akzeptablere Form der Zusammenarbeit zu erwirken: Hoover sollte öffentlich die Unabhängigkeit des AFSC bestätigen und einem eigenständigen fundraising des AFSC gemeinsam mit linken und liberalen Unterstützergruppen zustimmen. Hoover antwortete mit zwei gleichzeitig verfassten Schreiben, die sein zwiespältiges Verhältnis zu den Quäkern im Kontext der Russlandhilfe gut widerspiegelten: Einerseits leistete er Jones die gewünschte Erklärung, das AFSC genieße die volle Unterstützung der ARA, die russische Not zu lindern ,free of purpose in political, religious or racial contention“, und versicherte weiterhin, dass weder das AFSC noch andere am Unternehmen beteiligte Organisationen ,are in any way losing their identity or supervision of their own distribution subject only to coordination for the common good of the Russian people“. Die Aufgabe der ARA sei es, „to assure protection and efficiency in administration that every cent shall do its utmost in saving life“ sowie „that the whole effort shall be American in name and ideals“. ${ }^{88}$

Das zweite Schreiben schlug dagegen einen völlig anderen Ton an: Hoover mahnte Jones, nunmehr von weiteren Anfragen abzusehen und äußerte deutlichen Unmut darüber, dass die Frage der Unabhängigkeit des AFSC immer wieder von neuem aufgeworfen werde, offenbar „because a militant group of red-minded people are trying to undermine the ARA through the Friends Service Committee“. ${ }^{89}$ Jones wies in seiner Antwort ,any affiliation or leaning towards reds or pinks“ zurück und erklärte dem ,genuine and sincere friend“ Hoover die Loyalität des AFSC „to your great work“. Er versicherte, man werde „not tolerate for any instant any action on the part of the Service Committee (...) to injure you“". ${ }^{90}$ Mit dieser Erklärung wollte sich Hoover allerdings nicht zufrieden geben. Während er Jones’ persönliche Loyalität nicht in Frage stellte (,no-one has a higher appre-

87 Kenneth Durant an Wilbur Thomas 14/9/21, in: AFSCA, General Administration, Foreign Service, Country: Russia 1921 (fund raising letters).

88 Herbert Hoover an Rufus Jones 10/9/1921, in: General Administration, Foreign Service, Country: Russia 1921 (American Relief Administration).

89 Herbert Hoover an Rufus Jones 10/9/1921, in: AFSCA, General Administration, Foreign Service, Country: Russia 1921 (American Relief Administration).

90 Rufus Jones an Herbert Hoover 16/9/1921, in: AFSCA, General Administration, Foreign Service, Country: Russia 1921 (American Relief Administration). 
ciation of the singlemindedness of your own efforts than I have"), kritisierte er durchaus das AFSC, das doch durch seine Haltung den Erfolg der gesamten Hilfskampagne gefährde, weil der Eindruck der Uneinigkeit unter den Hilfsorganisationen erweckt werde. Ziel der radikalen Unterstützer sei es, „elements of discontent in this country under the cloak of relief“ $\mathrm{zu}$ streuen und dabei alle religiösen und bürgerlichen Elemente aus der russischen Hungerhilfe zu verdrängen. Man stehe auf derselben Seite und eine Zusammenarbeit mit der ARA sei folglich auch der beste Weg für die Quäker, „to be safe from being raided by the reds".91

Gereizt durch die Vielzahl der Angriffe aus der linken und liberalen Presse wurde Hoover in den folgenden Monaten immer ungehaltener über die Eingaben des AFSC. Bereits im November kam es zu einer weiteren Auseinandersetzung. Hintergrund war Jones' Bitte an Hoover, einen landesweiten Spendenaufruf des AFSC mittels einer öffentlichen Erklärung zu unterstützen. Das AFSC sah sich in Buzuluk außerstande, mit den zur Verfügung stehenden Mitteln die ihm zugewiesene Zahl von Hilfsbedürftigen (50.000 Personen) zu versorgen. Hoover verweigerte eine solche Erklärung. ${ }^{92}$

Doch nicht nur das: Nur kurze Zeit später veröffentlichte die ARA eine Pressemitteilung, in der sie bekannt gab, momentan seien keine weiteren Hilfslieferungen nach Russland angezeigt, da Hilfsgüter in ausreichendem Maße in den russischen Häfen lagerten und ohnehin Engpässe bei der Auslieferung bestünden. Diese Aussage schmälerte die Erfolgsaussichten eines Spendenaufrufs. Jones bat Hoover umgehend um die Rücknahme seiner Erklärung. Abermals lehnte Hoover ab. ${ }^{93}$

Damit war aus Sicht der Gegner Hoovers der Beweis erbracht, dass es der ARA vor allem darum ging, die Russlandhilfe zu monopolisieren und alle unabhängigen Organisationen ins Abseits zu drängen. Teile der Presse begannen Hoover nun „Sabotage“ der Quäkerhilfe vorzuhalten. Ein drittes Mal versuchte Jones daraufhin, Hoover zu einem Kurswechsel zu bewegen. ${ }^{94}$ Hoover bot an, eventuelle Ausfälle im Spendenaufkommen der Quäker durch ARA-Mittel auszugleichen,

91 Herbert Hoover an Rufus Jones 21/9/1921, in: AFSCA, Foreign Service Section, Country: Russia 1921 (Hoover correspondence).

92 Rufus Jones an Herbert Hoover o.D. (November/Dezember 1921), in: AFSCA, AFSCA, Foreign Service Section, Country: Russia 1921 (Hoover correspondence).

93 Rufus Jones an Herbert Hoover, Hoover an Jones o.D. (November/Dezember 1921), in: AFSCA, Foreign Service Section, Country: Russia 1921 (Hoover correspondence).

94 Rufus Jones an Herbert Hoover 4/1/1922, in: AFSCA, General Administration, Foreign Service, Country: Russia 1922 (Herbert Hoover). 
was wiederum all jene bestätigte, die Hoover politische Motive unterstellten. ${ }^{95}$ Hoover sah auch in der Folge immer wieder Anlass, sich über die „Hysterie“ linker Kreise und die Anwürfe aus dem Umfeld des AFSC zu beschweren. ${ }^{96}$ Er kritisierte das Komitee für seine indirekte Unterstützung eines Systems, das offen Atheismus propagiere: „I cannot conceive a greater negation of all that the Quakers stand for than a regime that carries on its banners ,Religion is the opiate of the people ${ }^{6}$ "“97

$\mathrm{Zu}$ einer Art showdown kam es im Februar 1922. Einige im AFSC wunderten sich über die „amazing campaign“ gegen die Arbeit der Quäker in Teilen der Hoover-freundlichen Presse. ${ }^{98}$ So berichtete Wilbur Thomas der Mission in Moskau von Presseberichten, die für ihn zweifelsfrei aus dem Umfeld der ARA kamen und nahelegten, die russischen Eisenbahnen seien nicht in der Lage, Hilfsgüter zu transportieren, was erneut eine schwere Hypothek für die Spendenkampagne in den USA bedeutete. ${ }^{99}$

Es häuften sich nun auch unter den Quäkern Stimmen, welche die offene Konfrontation mit Hoover suchen wollten. An William Albright in Moskau berichtete Wilbur Thomas von der erbitterten Stimmung im Komitee angesichts des Umstands, dass Hoovers Haltung ,has hurt our campaign very much. (...) Mr. Hoover's opinion carries great weight and when people begin to think that he has all the food that can be handled it does make a difference in our income." Gleichzeitig versäumten Hoover und andere ARA-Offizielle keine Gelegenheit, sich über die mangelnde Effizienz bei der Verteilung der Hilfsgüter seitens des AFSC zu beschweren. Thomas verwahrte sich wiederholt gegen die als rufschädigend empfundenen Vorwürfe und verlangte von Hoover eine Klarstellung. Als diese ausblieb, sprachen sich einige Mitglieder des AFSC nach Thomas' Darstel-

95 Herbert Hoover an Rufus Jones 6/1/1922, AFSCA, AFSCA, General Administration, Foreign Service, Country: Russia 1922 (Herbert Hoover), erneut und explizit findet sich das Angebot in einem Brief von Hoover an Rufus Jones 13/2/22, in AFSCA, General Administration, Foreign Service, Country: Russia 1922 (Herbert Hoover).

96 Herbert Hoover an Rufus Jones 6/1/1922, in: AFSCA, AFSCA, General Administration, Foreign Service, Country: Russia 1922 (Herbert Hoover). Gegenüber Paxton Hibben beschwerte sich Hoover, er sei es leid, von eben jenen Leuten, die ihm bereits vor Beginn der ARA-Aktion beständig seine Untätigkeit vorgeworfen hatten, nun weiterhin kritisiert zu werden,,because I do not do more or don’t do it in their way“. Hoover an Paxton Hibben 3/2/1922, in: AFSCA, AFSCA, General Administration, Foreign Service, Country: Russia 1922 (Herbert Hoover)

97 Wenn Jones Zweifel über die Ziele dieser Gruppen habe, so Hoover sarkastisch, „I would be glad to help you“. Herbert Hoover an Rufus Jones 13/2/1922, in: AFSCA, AFSCA, General Administration, Foreign Service, Country: Russia 1922 (Herbert Hoover).

98 Paxton Hibben (Medical Unit for Service in Russia) an Alfred Scattergood 10/2/1922, in: AFSCA, General Administration, Foreign Service, Country: Russia 1922 (Jane Addams).

99 Wilbur Thomas an Murray Kenworthy 9/2/1922, in: AFSCA, AFSCA, General Administration, Foreign Service, Country: Russia 1922 (correspondence from Philadelphia to Russia). 
lung für „very drastic action and refusal to have any further dealings with Mr. Hoover" aus. ${ }^{100}$

Ende Februar veröffentlichte dann der New York Call, eine sozialistische Zeitschrift, einen Artikel unter dem Titel „Hoover sabotages Russian relief“, der unter Berufung auf anonyme britische und amerikanische Quäker die ARA frontal angriff. Dieser Artikel brachte aus Hoovers Sicht das Fass endgültig zum Überlaufen. „I think the time has come when the Friends could take energetic measures to stop the propaganda constantly gotten out, that I have been endeavouring to embarrass them and retard their work“, schrieb er an Jones. Wenn Letzteres zutreffe, legte Hoover dem AFSC nahe, sei der sauberste Weg die Trennung. „If I or the ARA embarrass them, then the Friends should at once divorce themselves from all relations to the American Relief Administration to which I haven't the slightest objection or alternatively, they should put a stop to this nonsense. (...) If you have any feeling that you could be of greater service in this way, pray do what you think best!"101

An einem solchen Abbruch der Beziehungen hatte das AFSC, bei allen Schwierigkeiten im Verhältnis mit der ARA, jedoch kein Interesse, hätte dies doch das abrupte Ende der Aktion zur Folge gehabt. Wilbur Thomas versicherte Hoover, wenngleich nicht ohne eine gewisse Doppelbödigkeit, man wisse die Hilfe der ARA durchaus zu schätzen: „We deplore as you do this tendency to lose sight of the fundamental necessity to relieve starvation in Russia and to confuse the matter with personalities and opinions which are entirely beside the issue." ${ }^{\text {"102 }}$ Tatsächlich verlangte das AFSC im unmittelbaren Anschluss vom New York Call eine Gegendarstellung und warb gleichzeitig um Verständnis für die Position des AFSC, das von dem Ziel geleitet sei, zum frühestmöglichen Zeitpunkt zu einer Arbeitsweise zurückzukehren, die im Einklang „to our ideals and past practice“ stehe. ${ }^{103}$

100 Wilbur Thomas an William Albright 27/2/1922, in: AFSCA, General Administration, Foreign Service, Country: Russia 1922 (correspondence from Philadelphia to Russia).

101 Herbert Hoover an Rufus Jones 6/3/1922, in: AFSCA, General Administration, Foreign Service, Country: Russia 1922 (American Relief Administration).

102 Wilbur Thomas an Herbert Hoover 13/3/1922, in: AFSCA, General Administration, Foreign Service, Country: Russia 1922 (American Relief Administration).

103 Die getroffenen Übereinkünfte seien vorübergehender Natur und nur gegen die Zusicherung akzeptiert worden, dass diese nur für die Dauer der unmittelbaren Anwesenheit der ARA in Russland Gültigkeit besäßen, und dass die grundsätzlich eigenständige Identität der Quäkerhilfe gewahrt bleibe, versicherte Thomas. Auch die Anschuldigung, Hoover habe das fundraising des AFSC gezielt torpediert, die Thomas ja teilte, wurde zurückgewiesen, wenngleich mit dem Hinweis versehen, dass „time will decide“, ob Hoovers Behauptungen, Russland könne keine weiteren Hilfsgüter annehmen, der Wahrheit entsprächen. Das Schreiben schloss mit einem Bekenntnis zu 


\section{Selling „Red Relief“. Die Öffentlichkeitsarbeit in der russischen Hilfskampagne}

Vor dem Hintergrund dieser Streitigkeiten wird die gesteigerte Bedeutung der Öffentlichkeitsarbeit des AFSC während der russischen Kampagne deutlich. Wenn der Einsatz in der Sowjetunion für das AFSC einen weiteren wichtigen Schritt auf dem Weg zu einer etablierten Hilfsorganisation darstellte, so galt dies umso mehr für die Außendarstellung des Komitees. Wie auch für andere Hilfsorganisationen wie Save the Children oder das JDC wirkte die Russlandhilfe durch ihre besondere politische Brisanz speziell in diesem Bereich als Katalysator. ${ }^{104}$ Eine Vielzahl von Veröffentlichungen dokumentierte diesen Prozess. ${ }^{105}$ Vor der Übereinkunft mit Hoover ging es dem AFSC und seinen Unterstützern vor allem darum, einer dem Einsatz in der Sowjetunion gegenüber überwiegend kritischen, wenn nicht gar offen feindseligen Stimmung innerhalb der amerikanischen Öffentlichkeit entgegenzuwirken. Entscheidend war, den Eindruck zu zerstreuen, die Hilfe in Russland werde dazu beitragen, das bolschewistische Regime zu stabilisieren und ihm in der Auseinandersetzung mit seinen Gegnern einen strategischen Vorteil zu verschaffen. ${ }^{106}$ Anders formuliert: Das AFSC musste den Nachweis erbringen, dass die Hilfe tatsächlich bei den unmittelbar Betroffenen ankam und nicht etwa durch die Bolschewiki oder die Rote Armee zweckentfremdet wurde. ${ }^{107}$ Darüber hinaus bestand die eigentliche Herausforderung darin, ein positiveres Image der

Hoover, der mehr für die Arbeit der Quäker in Russland getan habe, „than any original plan contemplated“. Artikel wie der von Hoover zitierte seien in diesem Sinn ,damaging to the great cause for which we are all working“ und das AFSC sei folglich gezwungen, ihnen in der Öffentlichkeit in aller Schärfe entgegenzutreten, wolle es seine eigene Arbeit nicht gefährden. Wilbur Thomas an New York Call 26/3/1922, in: AFSCA, General Administration, Foreign Service, Country: Russia 1922 (Russia).

104 Linda Mahood and Vic Satzewich, „The Save the Children Fund and the Russian Famine of 1921-23: Claims and Counter-Claims About Feeding „Bolshevik“ Children,“ Journal of Historical Sociology 22, no. 1 (2009)., 55-83.

105 Milk, Soap and Fat for Russian Children, in: AFSC-Bulletin 40 (1921), One of the Great Disasters of History is Destroying the Child-Life of Russia, in: AFSC-Bulletin 41 (1921); Fighting the Famine in Russia, in: AFSC-Bulletin 41 (1921); Do you know the Reality of the Famine in Russia, in: AFSC-Bulletin 44 (1921); Thru the Valley of the Shadow of Death, in: AFSC-Bulletin 45 (1922); Does Russia need our help, in: AFSC-Bulletin 54 (1922); Death or Help from You, in: AFSC-Bulletin 57 (1923).

106 Die „general newspaper attitude is somewhat opposed“, hieß es bei Wilbur Thomas an Anna Haines 1/4/1921, in: AFSCA, General Administration, Foreign Service, Country: Russia 1921 (letters from Philadelphia to Russia)

107 Wilbur Thomas an Anna Haines 4/3/1921, in: General Administration, Foreign Service, Country: Russia 1921 (letters from Philadelphia to Russia). 
Sowjetregierung zu entwerfen, ohne dadurch den Verdacht zu erregen, zu deren Sympathisanten zu zählen. Es galt, die Hilfe in diesem Sinne in der öffentlichen Wahrnehmung so weit wie möglich zu entpolitisieren und den humanitären Charakter der Aktion herauszustreichen.

Ein wichtiges Element in dieser Hinsicht war - wie zuvor in Deutschland -, Kinder in den Mittelpunkt der Kampagne zu rücken: „It is not a question of politics, it is just a question of Saving Childrens’ Lives“, lautete die programmatische Überschrift über dem zentralen Spendenaufruf des AFSC. ${ }^{108}$ Ein Appell an die Quäker, der in den Gemeinden im Land verteilt wurde, schlug einen ähnlichen Ton an: „Ought children to be abandoned to the misery of slow starvation while men wrangle over politics, production and trade agreements(?)“, lautete die rhetorische Frage, an der, so der Text weiter, „rests the fate of millions of little children in Russia“. Bei anderer Gelegenheit wurde die „excellent work, that the Soviet Government is undertaking for its children“ gelobt. ${ }^{109}$ „No matter what one's individual feelings may be in regard to the Russian government“, hieß es in einem Aufruf im November 1921, „Christian America can not permit little children to starve when it is within their power to help them." ${ }^{110}$ Die beständige Wiederholung dieser Formeln lassen gleichzeitig erahnen, wie groß die Vorbehalte gegen die Arbeit in der Sowjetunion waren, auch und gerade im christlichen Amerika. Die Entpolitisierung und der Fokus auf die Kinder sowie der Versuch, die natürlichen Ursachen der Katastrophe ganz in den Vordergrund zu stellen, waren dafür die Indikatoren. Dass Kinder in den Mittelpunkt der Kampagne gerückt wurden, war umso auffälliger, als sie in Russland - anders als in Deutschland - nie die alleinige Zielgruppe der Speisungsaktionen waren. ${ }^{111}$

Auffällig in den Aufrufen des AFSC waren darüber hinaus die drastischen Schilderungen der Hungersnot und ihrer Auswirkungen auf die Kinder (und gelegentlich auf die Erwachsenen, meist in der Funktion als Eltern). Die Bilder unterschieden sich, was die eindringlichen Beschreibungen des Leidens betraf,

108 „It is not a question of politics, it is just a question of Saving Children’s Lives“ (AFSC-Leaflet) 14/11/1921, in: AFSCA, Foreign Service Section, Country: Russia 1921.

109 Wilbur Thomas an Anna Haines 9/2/1921, in: AFSCA, General Administration, Foreign Service, Country: Russia 1921 (letters from Philadelphia to Russia).

110 Spendenaufruf Wilbur Thomas 29/11/1921, in: AFSCA, Foreign Service Section, Country: Russia 1921 (Russia Fund Raising Letter)

111 Der Jahresbericht des AFSC für 1922 machte klar, dass die anfängliche Politik das Speisungsprogramm auf Kinder zu begrenzen, von der Realität rasch überholt worden war. „The feeding was extended to adults because it seemed essential to save adults as well as children and also because the feeding of children alone tended to break up homes and encourage parents to abandon their children“ AFSC Annual Report 1923, 11. 
auch deutlich von denen in Deutschland. Das Essen von Stroh oder Schuhleder, von Hunden und Tierleichen sowie Fälle von Kannibalismus waren wiederkehrende Topoi. Der Novemberaufruf des AFSC zitierte Anna Haines' Bericht aus Buzuluk, in dem sie von Leichen auf den Straßen und von Menschen berichtete, „eating indescribable food, cutting meat from their dead animals and making bread from grass, leaves and horses“ hoofs“, und von Waisenhäusern, „filled with wailing babies for whom there was no milk“. ${ }^{112}$

Solche persönlichen Schilderungen des Leids spielten eine große Rolle in der russischen Kampagne. Wilbur Thomas war darüber im permanenten Austausch mit der Hilfsmission in Moskau und Buzuluk und bemüht, geeignetes Material zu erhalten - insbesondere Bilder und Geschichten, die den individuell-menschlichen Aspekt hervorhoben -, um diese vielschichtige Strategie zu unterstützen. „Good pictures illustrating the life of people, pictures such as you like to look at in the illustrated papers“, waren gefragt, wie ein Schreiben an Anna Haines unterstrich. Ganz besonders galt es das Bild vom Elend der Kinder auf eine authentische, personalisierte sowie möglichst drastische Weise zu vermitteln: „To show the needs of the children it has proven necessary to have rather extreme cases, but if possible, they should not appear to be specially posed for the photograph but caught, as it were in the ordinary course of their daily life. A little story should accompany each picture (...).“An anderer Stelle hieß es hierzu nur knapp: „Pitiful but attractive children's pictures will be worth many times as much as they cost."113 Thomas war sich des Zynismus seiner immer wiederkehrenden Aufforderungen, solche Bilder mit einem ,poignant appeal“ zu liefern, durchaus bewusst, stellte jedoch gleichzeitig fest, es sei „difficult to draw money out of unwilling pockets without them“".114 Willkommen waren darüber hinaus auch Berichte, die halfen, „to break down the prejudice against the Soviet government“. ${ }^{115}$ Das AFSC zeigte sich zu diesem Zweck interessiert an den Aussagen von Russen unterschiedlicher politischer und religiöser Orientierung, von Intellektuellen und Wissenschaftlern, die bestätigten, dass „children of all classes are

112 Spendenaufruf Wilbur Thomas 29/11/1921, in: AFSCA, Foreign Service Section, Country: Russia 1921 (Russia Fund Raising Letter).

113 Wilbur Thomas an Anna Haines 30/6/1921, in: AFSCA, Foreign Service Section, Country: Russia 1921 (General).

114 Wilbur Thomas an Anna Haines 30/6/1921, in: AFSCA, Foreign Service Section, Country: Russia 1921 (General).

115 Wilbur Thomas an Anna Haines 1/4/1921, in: AFSCA, General Administration, Foreign Service, Country: Russia 1921 (letters from Philadelphia to Russia). 
treated without discrimination in the distribution of food by the Soviet Government as well as by the Friends“. ${ }^{116}$

Wenngleich die zentralen Topoi der Öffentlichkeitsarbeit konstant erhalten blieben, verschoben sich durch die Teilnahme des AFSC an der Kampagne der ARA einzelne Elemente oder erhielten ein anderes Gewicht. Der Einstieg der ARA im Sommer 1921 zog einen Teil der Aufmerksamkeit der Öffentlichkeit auf Hoover und unterstrich zum selben Zeitpunkt den Charakter der Quäkerhilfe als Teil einer US-amerikanischen Hilfsaktion. Wollten die Quäker ihren eigenen Anteil an der Spendenkampagne möglichst hochhalten, so musste das AFSC seine Öffentlichkeitsarbeit entsprechend diesem Ziel anpassen. ${ }^{117}$

Wilbur Thomas schwor die Moskauer Vertretung der Quäker vor diesem Hintergrund darauf ein, weiterhin Informationen zu liefern, die sich für die Spendenkampagnen zu Hause zielgenau verwenden ließen. Wenngleich sich die Mission in Russland auf die Hilfe selbst konzentrieren müsse, bat Thomas darum zu berücksichtigen, dass es die Darstellung dieser Hilfe gegenüber der Öffentlichkeit sei, die am Ende über den Erfolg entscheiden würde: „The right kind of service means more relief in the long run and more lives saved.“ In diesem Sinn bat Thomas Murray Kenworthy im April 1922 darum, im Moskauer Büro eigens für diesen Zweck eine Stelle zu schaffen, die den Informationsfluss koordinieren sollte, und zwar für eine Person, die fähig war, „to put the American letters in American words“. Eine Mehrzahl der Menschen in den USA denke nach wie vor, dass der überwiegende Teil der Hilfsgüter in die falschen, unbedürftigen, von der Regierung bestimmten Hände gerate. Gerade deshalb benötige man viele Berichte aus erster Hand, sowohl was den tatsächlichen Bedarf als auch was die Hilfsaktionen selbst betraf. Dazu gehörte auch, dass für den Moment der Eindruck vermieden werden musste, die Zusammenarbeit mit den Briten laufe unter der Hand weiter. $^{118}$

Die Appelle, der Öffentlichkeitsarbeit höchste Priorität einzuräumen, wurden im Laufe des Jahres 1922 nur noch dringlicher, nachdem die ARA im Frühjahr 1922 die Botschaft auszusenden begann, dass der Höhepunkt der Hungersnot überschritten sei. Auf diese Weise führte die russische Hilfsaktion zu einer funktionalen Differenzierung und Professionalisierung der Öffentlichkeitsarbeit des

116 Wilbur Thomas 29/4/1921 an , in: AFSCA, General Administration, Foreign Service, Country: Russia 1921 (letters from Philadelphia to Russia).

117 Wilbur Thomas an Arthur Watts 30/8/1921, in: AFSCA, General Administration, Foreign Service, Country: Russia 1921 (letters from Philadelphia to Russia).

118 Wilbur Thomas an Murray Kenworthy 12/4/1922, in: AFSCA, General Administration, Foreign Service, Country: Russia 1922 (correspondence from Philadelphia to Russia). 
AFSC. ${ }^{119}$ Das Material aus dem Feld wurde in Philadelphia für unterschiedliche Zwecke in Pressemitteilungen, Bulletins, Flugblättern und Informationsbroschüren aufbereitet. Der Pressedienst des AFSC bot dabei neben Erfahrungsberichten auch fertige Artikel zur Veröffentlichung in Quäkerpublikationen, aber auch in Zeitungen und Magazinen wie The New Republic oder National Geographic an. ${ }^{120}$ Daneben organisierte das AFSC Vortragsreisen einzelner Mitglieder der russischen Hilfsmission auf Heimaturlaub wie Murray Kenworthy oder Anna Haines. In vielen Staaten der USA gründeten sich lokale Russland-Komitees, die nicht nur die lokale Presse mit Erfahrungsberichten versorgten, sondern auch Pfarrer und Gemeindevorstände quer durch die USA dazu anhielten, die Hilfsaktionen in den Gottesdiensten publik zu machen. ${ }^{121}$

Die weitere Differenzierung der Öffentlichkeitsarbeit war damit ein bleibendes Erbe der russischen Hilfsaktion. Geschichten und Bilder waren dabei ein wichtiges Mittel, nicht nur um Spenden zu generieren. Es ging hierbei im Kern auch darum, den quäkerspezifischen Charakter der Hilfe hervorzuheben, der sich insbesondere in dem breiten Raum spiegelte, welcher der persönlichen Begegnung zwischen den Gebern und den Empfängern der Hilfe eingeräumt wurde. „The idea behind it will be that if one really knows others, he cannot hate them and that these glimpses of Russia have already helped to counteract a lot of American prejudice by arousing sympathy and appreciation in its place“, erklärte Thomas deren tiefere Bedeutung, als Ausdruck eines „Quakerly spirit““. ${ }^{122}$

Im Oktober 1922 schlossen die Quäker, nun wieder in einer gemeinsamen Mission vereint, ein neues Abkommen mit der Sowjetregierung, mit dem sie zunehmend von der Hunger- zur Wiederaufbauhilfe übergingen. Traktoren und Pferde wurden importiert, eine Reihe von Schulen und Kliniken errichtet und eine Landwirtschaftsschule in Totskoye unterhalten. ${ }^{123}$ Die Zeit von 1924 an gestaltete sich dann als eine Art Abschied auf Raten. Während im Hintergrund der Aufbau der Stalin'schen Diktatur voranschritt, verschlechterten sich zusehends die Be-

119 Wilbur Thomas an Walter Wildman (AFSC Moskau) 1/12/1922, in: AFSCA, General Administration, Foreign Service, Country: Russia 1922 (correspondence from Philadelphia to Russia). 120 Auch eigens produzierte Filme wurden eingesetzt. Siehe etwa einen kurzen Werbefilm aus dem Jahr 1922, der für die Weiterführung der Russlandaktion Spenden einsammeln wollte. AFSC, Archives: Friends Relief in Russia (https://youtu.be/BugMVBkmD6c, aufgerufen am 21.06. 2021). 121 In einem Rundbrief des AFSC wurden Kenworthy-Zitate mit der Bitte um Unterstützung der regionalen Presse verteilt. Auch wurden Pastoren in den Quäkergemeinden darum gebeten, den Aufruf in ihren Predigten bekannt zu machen. Rundbrief Wilbur Thomas 22/8/1922, in: AFSCA, Foreign Service, Country: Russia 1922.

122 Rundbrief Wilbur Thomas 22/8/1922, in: AFSCA, Foreign Service, Country: Russia 1922.

123 Siehe etwa The Consultation for Babies in Sorochinskoye, Samara/Helping Russian Mothers and Babies, in: AFSC-Bulletin 63 (1925); 
dingungen, unter denen die Quäker in der Sowjetunion arbeiteten. Die Partner der Quäker in Moskau und Buzuluk, allen voran die Tolstoianer, gerieten zunehmend unter Verfolgungsdruck, jede Art von religiöser Praxis unterlag strenger Beobachtung und Gängelung. Gleichzeitig verlor die Quäkermission schrittweise an Stärke. Nancy Babb kehrte nach dem abgeschlossenen Aufbau einer Geburtsklinik in Totskoye 1927 in die USA zurück. Als letzte Helferin verließ die irische Quäkerin Dorice White 1930 das Land und 1931 wurde auch das Quäkerzentrum in Moskau offiziell geschlossen. ${ }^{124}$

Russland bedeutete damit in vieler Hinsicht einen zweiten wichtigen Schritt in der humanitären Identitätsbildung des AFSC. Im Vergleich zu Deutschland stellte der Einsatz die Neutralität der Quäker in einer weiteren Hinsicht in Frage und zwang das Komitee in eine Debatte um den eigenen politischen Standpunkt. Mit wem, so lautete eine unterschwellige Frage, konnten die Quäker in den USA zusammenarbeiten, ohne den Konsens zu gefährden, für den die Idee des constructive service seit dem Ersten Weltkrieg eine Klammer darstellte. Der Einsatz hinterließ in dieser Hinsicht ein vielschichtiges Erbe. Die Politics of Neutrality waren hier erstmals bis an die äußerste Grenze getrieben worden, die Methoden der Öffentlichkeitsarbeit verfeinert. Für das Verhältnis und insbesondere die Zusammenarbeit mit den britischen Quäkern hatte der Einsatz einen weiteren Rückschlag gebracht und die ohnehin stotternden Pläne für eine umfassende internationale Kooperation zurückgeworfen. Aber auch in den USA selbst war das Erbe des russischen Einsatzes allgegenwärtig und floss, wie das folgende Kapitel zeigen wird, an vielen Stellen in die zukünftige Arbeit des Komitees ein.

\section{Exkurs: Das AFSC in Polen und Österreich}

Die Einsätze des AFSC in Deutschland und Russland waren Meilensteine in der Entwicklung einer Quäkerhilfsorganisation. Wenngleich die bei weitem größten und komplexesten, waren sie aber nicht die einzigen Hilfsoperationen, an denen sich amerikanische Quäker beteiligten. Von dem halben Dutzend kleiner Aktionen unter anderem in Serbien und Griechenland, ${ }^{125}$ an denen AFSC-Vertreter in un-

124 McFadden, „The Poltics of Relief: American Quakers and Russian Bolsheviks, 1917-1921,“ $16-17$.

125 Im Juli 1919 hatte das AFSC sechs Helfer zur Unterstützung des britischen Serbian Relief Fund nach Leskovatz in Zentral-Serbien geschickt. In der serbischen Hauptstadt Belgrad arbeitete eine kleine Anzahl von US-amerikanischen Quäkern von 1919 bis 1920 an der Seite einiger britischer Freunde neben der ARA. Barnes, A Centennial History of the American Friends Service Committee, 35-36. 
terschiedlicher Intensität und personeller Stärke teilnahmen, bieten insbesondere zwei - in Österreich und Polen - interessante Einblicke in die Entwicklung des Komitees zu einer dauerhaften Hilfsorganisation.

Eine besonders aufschlussreiche Perspektive eröffnete der Einsatz amerikanischer Quäker in Österreich - ganz besonders im Kontrast zu den zeitgleichen Kinderspeisungen in Deutschland. Auch das Zentrum des ehemaligen Habsburgerreichs gehörte wie Deutschland zu den Verlierern des Weltkriegs. Der österreichische Rumpfstaat, der aus den Trümmern der im Krieg untergegangenen K.u.-K.-Monarchie hervorgegangen war, befand sich nach 1918 sogar in einer noch prekäreren Situation als das Deutsche Reich. Abgeschnitten von großen Teilen der traditionellen Versorgungsgebiete in Ungarn, Böhmen, Polen und Galizien, war insbesondere die Hauptstadt Wien in einer verzweifelten Lage. Anders als in Deutschland waren die Sieger des Weltkriegs hier aber früh bereit, humanitäre Hilfsaktionen einzuleiten. Bereits im Frühjahr 1919, und damit noch während der Pariser Friedensverhandlungen, begann die ARA auf Herbert Hoovers Initiative hin mit einem Kinderhilfsprogramm in Österreich, das bis 1921 sukzessive ausgebaut wurde.

Zeitgleich errichteten auch die britischen Quäker im Mai 1919 eine Hilfsmission in Wien, die von Hilda Clark geleitet wurde, die damit unmittelbar an ihre Tätigkeit in Frankreich anschloss. An dieser Hilfsmission beteiligte sich das AFSC im Juli mit einigen ebenfalls in Frankreich erprobten Helfern. Die Leitung des AFSC-Kontingents übernahm Alfred Scattergoods Bruder Henry, der gleichzeitig der Europaleiter des AFSC war. Bis ins Frühjahr 1920 existierte in Wien eine Art Arbeitsteilung zwischen der großen und finanzstarken ARA-Hilfsorganisation, welche Kinder im Schulalter mit einer täglichen Mahlzeit versorgte, und der kleinen, angloamerikanischen Mission, die sich auf die Speisung von Kindern unter sechs Jahren sowie - wie in Deutschland - auf ein kleineres Studentenspeisungsprogramm konzentrierte. Die Gelder für diese Arbeit stammten aus privaten Spenden, wobei das AFSC für einen überproportional großen Anteil aufkam. ${ }^{126}$

Als Hoover im Mai 1920 für kurze Zeit Überlegungen anstellte, die ARA (bzw. der ECF) könnte ihre beziehungsweise seine Hilfsoperation in Österreich beenden

126 Franz Adlgasser, American Individualism Abroad: Herbert Hoover, Die American Relief Administration Und Österreich, 1919-1923 (Wien: VWGÖ, 1993). Hierzu auch A. Ruth Fry, A Quaker Adventure: The Story of Nine Years' Relief and Reconstruction (London: Nisbet \& Co., 1926), 192244; Religiöse Gesellschaft der Freunde (Hg.), Wege zur Versöhnung: Humanitäre Hilfe der Quäker in Österreich. Begleitheft zur Ausstellung anlässlich des 50. Jahrestags der Verleihung des Friedensnobelpreises an die Quäker (Wien: Ausstellungskomitee der Religiösen Gesellschaft der Freunde, 1997). 
und alle Hilfe in ein gemeinsames deutsch-polnisch-österreichisches Hilfswerk unter Leitung des AFSC überführen, stand abermals die Frage der „Amerikanisierung“ der Quäkerhilfe und folglich einer Trennung der angloamerikanischen Mission im Raum. Dass es dazu nicht kam, lag vor allem am Widerstand der ARAVertreter in Wien. Deren Leiter Gilchrist Stockton störte sich neben der religiösen Ausrichtung der Quäker vor allem an deren generellem Hilfsansatz, welcher aus seiner Sicht von dem durchweg rationalen und effizienten der ARA abwich. Die Quäker, die Stockton offenbar mit der britischen Leitung identifizierte, legten nach seiner Beobachtung zu großen Wert auf persönliche Begegnungen als Teil der Hilfe und hatten aus diesem Grund einen vergleichsweise hohen Personalaufwand - anders als die ARA, die mit wenigen amerikanischen Helfern und hauptsächlich mit einheimischem Personal operierte. Schließlich wurde die angloamerikanische Mission in Wien nach Einschätzung Stocktons von den Österreichern in erster Linie als britisches Unternehmen wahrgenommen - in der Tat standen 40 Briten 14 Amerikanern gegenüber - was mit dem Ziel einer rein amerikanischen Hilfsaktion nicht $\mathrm{zu}$ vereinbaren war. ${ }^{127}$

Im Gegensatz zu Stockton waren die Vertreter des AFSC hocherfreut über Hoovers anfängliche Idee, die Kinderspeisungsaktion in Wien unter Quäker-Regie zu nehmen. Henry Scattergood machte umgehend klar, dass das AFSC zur Verfügung stand und - sofern dies die Trennung von den britischen Quäkern und die vorübergehende Auflösung der „internationalen“ Mission erforderte - dass das AFSC diesen Schritt umgehend und ohne Zögern zu vollziehen bereit war. Am Ende kam es anders und die ARA bzw. der ECF entschied, die Aktion bis Juni 1922 doch in Eigenregie weiterzuführen. Nach 1921 band die ARA dann das AFSC in Teile der Kinderspeisungsaktion ein - als rein amerikanisches Unternehmen, unter amerikanischer Flagge und folglich gänzlich ohne britische Beteiligung. ${ }^{128}$

Was bei dieser Episode abermals deutlich wurde: Im Zweifelsfall zogen die Vertreter des AFSC auch in Wien die Zusammenarbeit mit der ARA einer Praxis der

127 Zur ARA-Aktion vor allem Adlgasser, American Individualism Abroad: Herbert Hoover, Die American Relief Administration Und Österreich, 1919-1923. Adlgasser betont den dezidiert amerikanischen Ton der Kampagne, der noch deutlicher ausfiel als in Deutschland. Die sogenannten Hyphenate-Americans, in diesem Fall Austro-Amerikaner, wurden auch hier eingebunden - allerdings durch die ARA selbst.

128 Der Austausch zwischen Gilchrist Stockton und Scattergood bei Adlgasser, American Individualism abroad, 135-141. 
reinen Lehre des Quaker relief in Zusammenarbeit mit ihren britischen Glaubensgenossen vor. ${ }^{129}$

Aufschlüsse eigener Art bietet dagegen der Einsatz in Polen. ${ }^{130}$ Hier erlebte die kleine Gruppe von AFSC-Helfern 1919 die „most appalling conditions of need with which our workers have been in touch“. ${ }^{131}$ Sie trafen auf ein Land, das ähnlich den russischen Hungergebieten, vom Krieg und den nachfolgenden politischen Verwerfungen schwer verwüstet worden war. Seit 1914 hatte das polnische Territorium eine Fülle von Flüchtlingsbewegungen erlebt, die dem Kriegsverlauf sowie den Grenzziehungen und -konflikten nach Schaffung eines polnischen Staates in Versailles gefolgt waren. Im Sommer 1919 begann das AFSC an der Seite eines britischen Kontingents seine Arbeit, die sich in den beiden südgalizischen Bezirken Zawiercie und Nadworna (in der heutigen West-Ukraine) konzentrierte. Stand zu Anfang der Kampf gegen die grassierende Typhus-Epidemie im Mittelpunkt, so wandten die Quäker ihre Aufmerksamkeit bald einer Reihe von weiteren Aktivitäten zu, insbesondere in der Kinderhilfe, der Arbeit mit den in den Bezirken konzentrierten Flüchtlingen sowie der Hilfe für Universitätsstudenten. Auffallend an all diesen Aktivitäten war, dass der ,internationale“, sprich: angloamerikanische Charakter der Quäkermission hier offensichtlich kein Problem darstellte, und dies obwohl das AFSC offiziell an der Seite der ARA und des Amerikanischen Roten Kreuzes ins Land gekommen war. ${ }^{132}$ Ein Grund war unter Umständen, dass das kleine amerikanische Kontingent von rund zehn Personen im Vergleich zum 40-köpfigen britischen Kontingent kaum ins Gewicht fiel, die Quäker jedoch aus Sicht der ARA, die Polen nicht zuletzt als anti-bolschewistischen Frontstaat zu unterstützen hofften, eine wichtige Funktion er-

129 Einen Überblick über den österreichischen Einsatz bietet Work of Friends Relief Mission in Austria, in: AFSC-Bulletin 32 (1920); Quaker Relief Work in Vienna, 1922-1923, in: AFSC-Bulletin 55 (1923).

130 Einen Überblick der ersten Jahre des Einsatzes findet sich in der Broschüre Two years in Poland, in: AFSC-Bulletin 42(1921). Persönliche Erfahrungsberichte zu den frühen Jahren Susan Pares, Displaced by War: Getrude Powicke and Quaker Relief in France and Poland, 1915-1919 (London: Francis Boutle Publishers, 2015).; oder mit Schwerpunkt auf den britischen Teil der Mission: Jane Cooper, Embroidering History. An Englishwoman's Experience as a Humanitarian Aid Volunteer in Post-War Poland, 1924-1925 (Derby: DB Publishing, 2012). Zum breiteren Kontext ohne besondere Schwerpunktsetzung auf die Quäker Davide Rodogno, Francesca Piana, and Shaloma Gauthier, „Shaping Poland: Relief and Rehabilitation Programs by Foreign Organizations, 1918-1922," in Shaping the Transnational Sphere: Experts, Networks , and Issues from the 1840s to the 1930s, ed. Davide Rodogno, Bernhard Struck, and Jakob Vogel (Oxford: Berghahn Books, 2014).

131 Conditions in Eastern and Central Europe, in: AFSC-Bulletin 34 (1920).

132 The Society of Friends in Poland, in: AFSC-Bulletin 36 (1920). 
füllten. Auf der anderen Seite fand der internationale Charakter der Quäkermission in Polen, und erst recht die britische Dominanz, in der Darstellung des AFSC nach außen praktisch keine Erwähnung.

Überhaupt zeichnete sich der polnische Einsatz gerade dadurch aus, dass in den Berichten etwaige quäkerspezifische Züge der Hilfsaktion ausgeblendet waren und auch darüber hinaus, wenn überhaupt, eine vollständig untergeordnete Rolle spielten. Im Gegenteil: Das AFSC trat hier als eine in jeder Hinsicht streng nach rationalen Kriterien operierende Hilfsorganisation auf, deren einziges Ziel die Rettung von Menschenleben war. Statt persönlicher Begegnungen mit den Hilfebedürftigen oder des Versöhnungsaspekts wurden ganz die Erfolge der Hilfe selbst in den Vordergrund gestellt, etwa bei der Verbreitung von „ideas of cleanliness“ unter der lokalen Bevölkerung. ${ }^{133}$ Das AFSC handele in Polen, so hieß es in einem Artikel im AFSC-Bulletin 1921, „largely as a service organization“. Um diesen Dienst leisten zu können, unterhalte man eine Hilfsmission aus „carefully selected persons, doctors, nurses, trained social workers, agriculturalists and child welfare workers“.

Eine prominente Position nahmen auch Verweise auf den aktivierenden Charakter der Hilfe ein, im Sinne einer „Hilfe zur Selbsthilfe“, die ganz im Einklang mit den von der ARA vertretenen Prinzipien standen. ${ }^{134}$ Die Politik des AFSC sei es, ,to help local organizations to get back to self-support and efficiency and then to leave them to their local management“. Betont wurde häufig der vorübergehende Charakter der Aktionen sowie die Würdigkeit und Bereitschaft der Empfänger, die Hilfe zu nutzen, um wieder auf eigenen Beinen zu stehen. Dies erfüllte auch den Zweck, dem Vorwurf der Ineffizienz und zu breiter Streuung der Hilfe des AFSC zu begegnen (sowohl in Polen selbst, aber auch mit Blick auf die Vielzahl der parallelen Aktionen). ${ }^{135}$ Wenn es gleichzeitig überhaupt Ansätze zu message work in Polen gab, so konzentrierten sich diese auf Warschau und vorwiegend auf die Arbeit mit der Studenten- und der Mittelstandshilfe. Wie sich im Lauf der Zeit zeigen sollte, hatten die Quäker im tiefkatholischen Polen einen

133 The Society of Friends in Poland, in: AFSC-Bulletin 36 (1920).

134 Hierzu hieß es: „Help of every kind is needed to reestablish them (refugees) and this is a form of help which is especially worth giving as these who receive are not beggars but may become in a short time self supporting and self respecting peasants even repaying in part the help given them.“ In: The Society of Friends in Poland, in: AFSC-Bulletin 36 (1920).

135 The Society of Friends in Poland, in: AFSC-Bulletin 36 (1920). 
schweren Stand, so dass selbst diese zarten Ansätze kaum dauerhafte Früchte trugen. ${ }^{136}$

Polen bot damit eine Variante des grundsätzlichen Dilemmas, mit dem sich das AFSC in all seinen Einsätzen konfrontiert sah: Je professioneller das Komitee als Hilfsorganisation auftrat und sich etwa in seinem Stil an die ARA anglich, desto mehr drohten der quäkerspezifische Gehalt der Hilfe und die tieferliegenden Ziele, die sich mit der Hilfe verbanden, aus dem Blickfeld zu geraten. Polen war damit eines der Einsatzgebiete, das Fragen aufwarf, die das AFSC immer begleitet hatten, die nun aber, Mitte der 1920er Jahre, nach dem Ende der großen Nachkriegseinsätze, auf eine Antwort drängten.

136 Über die Arbeit nach dem Ende der Hilfsmission: The Return of the Poles from Exile, in: AFSC-Bulletin 50 (1922); The Anglo-American Mission of the Society of Friends in Poland, in: AFSCBulletins 70 (1926). Bis 1930 bestand ein Quäkerzentrum in Warschau weiter. 